\title{
BLM realization for Frobenius-Lusztig Kernels of type $A$
}

\author{
QIANG FU
}

The infinitesimal quantum $\mathfrak{g l}_{n}$ was realized in $[1, \S 6]$. We will realize Frobenius-Lusztig kernels of type $A$ in this paper.

\section{Introduction}

In 1990, Ringel discovered the Hall algebra realization [21] of the positive part of the quantum enveloping algebras of finite type. Almost at the same time, the entire quantum $\mathfrak{g l}_{n}$ was realized by A. A. Beilinson, G. Lusztig and R. MacPherson in [1]. They first used $q$-Schur algebras to construct a $\mathbb{Q}(v)$-algebra $\widehat{\mathcal{K}}_{\mathcal{Q}}(n)$, and then proved that the quantum enveloping algebra of $\mathfrak{g l}_{n}$ over $\mathbb{Q}(v)$ can be realized as a subalgebra of $\widehat{\mathcal{K}}_{\mathcal{Q}}(n)$.

Let $U_{k}(n)$ be the the quantum enveloping algebra of $\mathfrak{g l}_{n}$ over $\mathcal{K}$ with standard generators $E_{i}^{(m)}, F_{i}^{(m)}, K_{i}^{ \pm 1}$ and $\left[\begin{array}{c}K_{i} ; 0 \\ t\end{array}\right]$, where $\mathcal{K}$ is a commutative ring containing a primitive $l^{\prime}$ th root $\varepsilon$ of 1 . Let $p=\operatorname{char} k$. For $h \geqslant 1$, let $\widetilde{\mathrm{u}}_{\kappa}(n)_{h}$ be the $\mathcal{K}$-subalgebra of $U_{k}(n)$ generated by $E_{i}^{(m)}, F_{i}^{(m)}, K_{j}^{ \pm 1},\left[\begin{array}{c}K_{j} ; 0 \\ t\end{array}\right]$ for $1 \leqslant i \leqslant n-1,1 \leqslant j \leqslant n$ and $0 \leqslant m, t<l p^{h-1}$, where $l=l^{\prime}$ if $l^{\prime}$ is odd, and $l=l^{\prime} / 2$ otherwise. Then we have $\widetilde{\mathrm{u}}_{k}(n)_{1} \subseteq \widetilde{\mathrm{u}}_{k}(n)_{2} \subseteq \cdots \subseteq U_{k}(n)$. In the case where $l^{\prime}$ is an odd number, let $\mathrm{u}_{k}(n)_{h}=\widetilde{\mathrm{u}}_{\mathcal{k}}(n)_{h} /\left\langle K_{1}^{l}-1, \ldots, K_{n}^{l}-1\right\rangle$. The algebra $\mathfrak{u}_{\kappa}(n)_{1}$ is called the infinitesimal quantum $\mathfrak{g l}_{n}$ and the algebra $\mathrm{u}_{k}(n)_{h}$ is called Frobenius-Lusztig kernels of $U_{k}(n)$ (cf. [7]). The algebra $\mathrm{u}_{k}(n)_{1}$ was realized in $[1, \S 6]$. In this paper, we will realize the algebra $\mathrm{u}_{\kappa}(n)_{h}$ for all $h \geqslant 1$. More precisely, we will first construct the $\kappa$-algebra $\mathscr{K}^{\prime}(n)_{h}$ in $\S 4$. Then we will prove in 5.5 that $\mathrm{u}_{\kappa}(n)_{h} \cong \mathscr{K}^{\prime}(n)_{h}$ in the case where $l^{\prime}$ is odd, and that $\widetilde{\mathrm{u}}_{k}(n)_{h} \cong \mathscr{K}^{\prime}(n)_{h}$ in the case where $l^{\prime}$ is even and $\kappa$ is a field.

Let $\mathcal{S}_{k}(n, r)$ be the $q$-Schur algebra over $\mathcal{K}$. A certain subalgebra, denoted by $\widetilde{\mathrm{u}}_{\kappa}(n, r)_{h}$, of $\mathcal{S}_{k}(n, r)$ was constructed in [12, §4]. It is proved in [13] that $\widetilde{\mathrm{u}}_{\kappa}(n, r)_{1}$ is isomorphic to the little $q$-Schur algebra introduced in $[11,14]$. We will prove in 6.1 that the algebra $\widetilde{\mathrm{u}}_{k}(n, r)_{h}$ is a homomorphic image of $\widetilde{\mathrm{u}}_{\kappa}(n)_{h}$. 
Infinitesimal $q$-Schur algebras are certain important subalgebras of $q$ Schur algebras (cf. $[2,3,6]$ ). For $h \geqslant 1$ let $\mathbf{s}_{k}(n)_{h}$ be the $k$-subalgebra of $U_{k}(n)$ generated by the algebra $\widetilde{\mathrm{u}}_{k}(n)_{h}$ and $\left[\begin{array}{c}K_{j} ; 0 \\ t\end{array}\right](1 \leqslant j \leqslant n, t \in \mathbb{N})$. We will prove in 6.4 that the infinitesimal $q$-Schur algebra $\mathbf{s}_{k}(n, r)_{h}$ is a homomorphic image of $\mathbf{s}_{k}(n)_{h}$.

Throughout this paper, let $\mathcal{Z}=\mathbb{Z}\left[v, v^{-1}\right]$ where $v$ is an indeterminate and let $\mathcal{Q}=\mathbb{Q}(v)$ be the fraction field of $\mathcal{Z}$. For $i \in \mathbb{Z}$ let $[i]=\frac{v^{i}-v^{-i}}{v-v^{-1}}$. For integers $N, t$ with $t \geqslant 0$, let

$$
\left[\begin{array}{c}
N \\
t
\end{array}\right]=\frac{[N][N-1] \cdots[N-t+1]}{[t]^{!}} \in \mathcal{Z}
$$

where $[t]^{!}=[1][2] \cdots[t]$. For $\mu \in \mathbb{Z}^{n}$ and $\lambda \in \mathbb{N}^{n}$ let $\left[\begin{array}{c}\mu \\ \lambda\end{array}\right]=\left[\begin{array}{c}\mu_{1} \\ \lambda_{1}\end{array}\right] \cdots\left[\begin{array}{c}\mu_{n} \\ \lambda_{n}\end{array}\right]$. For $\lambda, \mu \in \mathbb{Z}^{n}$, write $\lambda \leqslant \mu \Leftrightarrow \lambda_{i} \leqslant \mu_{i}$ for $1 \leqslant i \leqslant n$. We say that $\lambda<\mu$ if $\lambda \leqslant \mu$ and $\lambda \neq \mu$.

Let $\kappa$ be a commutative ring containing a primitive $l^{\prime}$ th root $\varepsilon$ of 1 with $l^{\prime} \geqslant 1$. Let $l \geqslant 1$ be defined by

$$
l= \begin{cases}l^{\prime} & \text { if } l^{\prime} \text { is odd }, \\ l^{\prime} / 2 & \text { if } l^{\prime} \text { is even } .\end{cases}
$$

Let $p$ be the characteristic of $\kappa$. We will regard $\mathcal{K}$ as a $\mathcal{Z}$-module by specializing $v$ to $\varepsilon$. When $v$ is specialized to $\varepsilon,\left[\begin{array}{c}c \\ t\end{array}\right]$ specialize to the element $\left[\begin{array}{c}c \\ t\end{array}\right]_{\varepsilon}$ in $k$.

\section{The BLM construction of quantum $\mathfrak{g l}_{n}$}

Following [17] we define the quantum enveloping algebra $U_{\mathcal{Q}}(n)$ of $\mathfrak{g l}_{n}$ to be the $\mathbb{Q}(v)$-algebra with generators

$$
E_{i}, F_{i} \quad(1 \leqslant i \leqslant n-1), K_{j}, K_{j}^{-1} \quad(1 \leqslant j \leqslant n)
$$

and relations

(a) $K_{i} K_{j}=K_{j} K_{i}, K_{i} K_{i}^{-1}=1$;

(b) $K_{i} E_{j}=v^{\delta_{i, j}-\delta_{i, j+1}} E_{j} K_{i}$;

(c) $K_{i} F_{j}=v^{\delta_{i, j+1}-\delta_{i, j}} F_{j} K_{i}$;

(d) $E_{i} E_{j}=E_{j} E_{i}, F_{i} F_{j}=F_{j} F_{i}$ when $|i-j|>1$;

(e) $E_{i} F_{j}-F_{j} E_{i}=\delta_{i, j} \frac{\widetilde{K}_{i}-\widetilde{K}_{i}^{-1}}{v-v^{-1}}$, where $\widetilde{K}_{i}=K_{i} K_{i+1}^{-1}$;

(f) $E_{i}^{2} E_{j}-\left(v+v^{-1}\right) E_{i} E_{j} E_{i}+E_{j} E_{i}^{2}=0$ when $|i-j|=1$;

(g) $F_{i}^{2} F_{j}-\left(v+v^{-1}\right) F_{i} F_{j} F_{i}+F_{j} F_{i}^{2}=0$ when $|i-j|=1$. 
Following [19], let $U_{\mathcal{Z}}(n)$ be the $\mathcal{Z}$-subalgebra of $U_{\mathcal{Q}}(n)$ generated by all $E_{i}^{(m)}, F_{i}^{(m)}, K_{i}^{ \pm 1}$ and $\left[\begin{array}{c}K_{i} ; 0 \\ t\end{array}\right]$, where for $m, t \in \mathbb{N}$,

$$
E_{i}^{(m)}=\frac{E_{i}^{m}}{[m] !}, \quad F_{i}^{(m)}=\frac{F_{i}^{m}}{[m] !}, \quad \text { and } \quad\left[\begin{array}{c}
K_{i} ; 0 \\
t
\end{array}\right]=\prod_{s=1}^{t} \frac{K_{i} v^{-s+1}-K_{i}^{-1} v^{s-1}}{v^{s}-v^{-s}}
$$

Let $\Theta(n)$ be the set of all $n \times n$ matrices over $\mathbb{N}$. Let $\Theta^{ \pm}(n)$ be the set of all $A \in \Theta(n)$ whose diagonal entries are zero. Let $\Theta^{+}(n)$ (resp. $\left.\Theta^{-}(n)\right)$ be the subset of $\Theta(n)$ consisting of those matrices $\left(a_{i, j}\right)$ with $a_{i, j}=0$ for all $i \geqslant j$ (resp. $i \leqslant j$ ). For $A \in \Theta^{ \pm}(n)$, write $A=A^{+}+A^{-}$with $A^{+} \in \Theta^{+}(n)$ and $A^{-} \in \Theta^{-}(n)$. For $A \in \Theta^{ \pm}(n)$ let

$$
E^{\left(A^{+}\right)}=\prod_{\substack{i \leqslant s<j \\ 1 \leqslant i, j \leqslant n}} E_{s}^{\left(a_{i j}\right)}, \quad F^{\left(A^{-}\right)}=\prod_{\substack{j \leqslant s<i \\ 1 \leqslant i, j \leqslant n}} F_{s}^{\left(a_{i, j}\right)}
$$

where the ordering of the products is the same as in $[1,3.9]$. According to $[19,4.5]$ and $[20,7.8]$ we have the following result.

Proposition 2.1. The set

$$
\left\{E^{\left(A^{+}\right)} \prod_{1 \leqslant i \leqslant n} K_{i}^{\delta_{i}}\left[\begin{array}{c}
K_{i} ; 0 \\
\lambda_{i}
\end{array}\right] F^{\left(A^{-}\right)} \mid A \in \Theta^{ \pm}(n), \delta, \lambda \in \mathbb{N}^{n}, \delta_{i} \in\{0,1\}, \forall i\right\}
$$

forms a $\mathcal{Z}$-basis of $U_{\mathcal{Z}}(n)$.

Using the stabilization property of the multiplication of $q$-Schur algebras, an important algebra $\mathcal{K}_{\mathcal{Z}}(n)$ over $\underset{\mathcal{Z}}{\mathcal{U}}$ (without 1 ), with basis $\{[A] \mid A \in \widetilde{\Theta}(n)\}$ was constructed in $[1,4.5]$, where $\widetilde{\Theta}(n)=\left\{\left(a_{i j}\right) \in M_{n}(\mathbb{Z}) \mid a_{i j} \geqslant 0 \forall 1 \leqslant i \neq\right.$ $j \leqslant n\}$. It should be noted that the algebra $\mathcal{K}_{\mathcal{Z}}(n)$ is isomorphic to the Lusztig integral form of the modified quantum $\mathfrak{g l}_{n}$ (cf. [10,6.3]).

For $A \in \widetilde{\Theta}(n)$ let $\operatorname{ro}(A)=\left(\sum_{1 \leqslant j \leqslant n} a_{1, j}, \ldots, \sum_{1 \leqslant j \leqslant n} a_{n, j}\right)$ and $\operatorname{co}(A)=$ $\left(\sum_{1 \leqslant i \leqslant n} a_{i, 1}, \ldots, \sum_{1 \leqslant i \leqslant n} a_{i, n}\right)$. Note that if $A, B \in \widetilde{\Theta}(n)$ is such that $\operatorname{co}(B) \neq \operatorname{ro}(A)$ then $[B] \cdot[A]=0$ in $\mathcal{K}_{\mathcal{Z}}(n)$. For $1 \leqslant i, j \leqslant n$ let $E_{i, j}$ be the $n \times n$ matrix whose $i, j$ entry is 1 and all other entries are zero. For $r \in \mathbb{N}$ let $\Lambda(n, r)=\left\{\lambda \in \mathbb{N}^{n} \mid \sum_{1 \leqslant i \leqslant n} \lambda_{i}=r\right\}$. From [1, 4.6(c)] we see that the algebra $\mathcal{K}_{\mathcal{Z}}(n)$ is generated by the elements $\left[m E_{i, i+1}+\operatorname{diag}(\lambda)\right],\left[m E_{i+1, i}+\operatorname{diag}(\lambda)\right]$ for $m \in \mathbb{N}, 1 \leqslant i \leqslant n-1$ and $\lambda \in \mathbb{Z}^{n}$. Furthermore the following multiplication formulas in $\mathcal{K}_{\mathcal{Z}}(n)$ is given in $[1,4.6(\mathrm{a}),(\mathrm{b})]$.

Proposition 2.2. Assume that $1 \leqslant i \leqslant n-1, m \in \mathbb{N}$ and $A \in \widetilde{\Theta}(n)$. 
(1) If $B \in \widetilde{\Theta}(n)$ is such that $\operatorname{co}(B)=\operatorname{ro}(A)$ and $B-m E_{i, i+1}$ is a diagonal matrix, then

$$
[B] \cdot[A]=\sum_{\substack{\mathbf{t} \in \Lambda(n, m) \\
\forall u \neq i+1, t_{u} \leqslant a_{i+1, u}}} v^{\beta(\mathbf{t}, A)} \prod_{1 \leqslant u \leqslant n}\left[\begin{array}{c}
a_{i, u}+t_{u} \\
t_{u}
\end{array}\right]\left[A+\sum_{1 \leqslant u \leqslant n} t_{u}\left(E_{i, u}^{\Delta}-E_{i+1, u}^{\Delta}\right)\right],
$$

where $\beta(\mathbf{t}, A)=\sum_{j>u}\left(a_{i, j}-a_{i+1, j}\right) t_{u}+\sum_{u<u^{\prime}} t_{u} t_{u^{\prime}}$.

(2) If $C \in \widetilde{\Theta}(n)$ is such that $\operatorname{co}(C)=\operatorname{ro}(A)$ and $C-m E_{i+1, i}$ is a diagonal matrix, then

$$
\begin{gathered}
{[C] \cdot[A]=\sum_{\substack{\mathbf{t} \in \Lambda(n, m) \\
\forall u \neq i, t_{u} \leqslant a_{i, u}}} v^{\gamma(\mathbf{t}, A)} \prod_{1 \leqslant u \leqslant n}\left[\begin{array}{c}
a_{i+1, u}+t_{u} \\
t_{u}
\end{array}\right]\left[A-\sum_{1 \leqslant u \leqslant n} t_{u}\left(E_{i, u}^{\Delta}-E_{i+1, u}^{\Delta}\right)\right],} \\
\text { where } \gamma(\mathbf{t}, A)=\sum_{j<u}\left(a_{i+1, j}-a_{i, j}\right) t_{u}+\sum_{u<u^{\prime}} t_{u} t_{u^{\prime}} .
\end{gathered}
$$

Following $[1,5.1]$, let $\widehat{\mathcal{K}}_{\mathcal{Q}}(n)$ be the vector space of all formal $\mathbb{Q}(v)$ linear combinations $\sum_{A \in \widetilde{\Theta}(n)} \beta_{A}[A]$ satisfying the following property: for any $\mathbf{x} \in \mathbb{Z}^{n}$,

$$
\text { the sets } \begin{aligned}
& \left\{A \in \widetilde{\Theta}(n) \mid \beta_{A} \neq 0, \operatorname{ro}(A)=\mathbf{x}\right\} \\
& \left\{A \in \widetilde{\Theta}(n) \mid \beta_{A} \neq 0, \operatorname{co}(A)=\mathbf{x}\right\}
\end{aligned} \text { are finite. }
$$

The product of two elements $\sum_{A \in \widetilde{\Theta}(n)} \beta_{A}[A], \sum_{B \in \widetilde{\Theta}(n)} \gamma_{B}[B]$ in $\widehat{\mathcal{K}}_{\mathcal{Q}}(n)$ is defined to be $\sum_{A, B} \beta_{A} \gamma_{B}[A] \cdot[B]$ where $[A] \cdot[B]$ is the product in $\mathcal{K}_{\mathcal{Z}}(n)$. Then $\widehat{\mathcal{K}}_{\mathcal{Q}}(n)$ becomes an associative algebra over $\mathbb{Q}(v)$.

For $A \in \Theta^{ \pm}(n), \delta \in \mathbb{Z}^{n}$ and $\lambda \in \mathbb{N}^{n}$ let

$$
\begin{aligned}
A(\delta, \lambda) & =\sum_{\mu \in \mathbb{Z}^{n}} v^{\mu \cdot \delta}\left[\begin{array}{l}
\mu \\
\lambda
\end{array}\right][A+\operatorname{diag}(\mu)] \in \widehat{\mathcal{K}}_{\mathcal{Q}}(n) ; \\
A(\delta) & =\sum_{\mu \in \mathbb{Z}^{n}} v^{\mu \cdot \delta}[A+\operatorname{diag}(\mu)] \in \widehat{\mathcal{K}}_{\mathcal{Q}}(n),
\end{aligned}
$$

where $\mu \cdot \delta=\sum_{1 \leqslant i \leqslant n} \mu_{i} \delta_{i}$.

The next result is proved in $[1,5.5,5.7]$. 
Theorem 2.3. There is an injective algebra homomorphism $\varphi: U_{\mathcal{Q}}(n) \rightarrow$ $\widehat{\mathcal{K}}_{\mathcal{Q}}(n)$ satisfying

$$
E_{i} \mapsto E_{i, i+1}(\mathbf{0}), K_{1}^{j_{1}} K_{2}^{j_{2}} \cdots K_{n}^{j_{n}} \mapsto 0(\mathbf{j}), F_{i} \mapsto E_{i+1, i}(\mathbf{0})
$$

Furthermore the set $\left\{A(\mathbf{j}) \mid A \in \Theta^{ \pm}(n), \mathbf{j} \in \mathbb{Z}^{n}\right\}$ forms a $\mathbb{Q}(v)$-basis for $\varphi\left(U_{\mathcal{Q}}(n)\right)$.

We shall identify $U_{\mathcal{Q}}(n)$ with $\varphi\left(U_{\mathcal{Q}}(n)\right)$. According to [16, 4.2,4.3,4.4], we have the following result.

Proposition 2.4. The algebra $U_{\mathcal{Z}}(n)$ is generated as a $\mathcal{Z}$-module by the elements $A(\delta, \lambda)$ for $A \in \Theta^{ \pm}(n), \delta \in \mathbb{Z}^{n}$ and $\lambda \in \mathbb{N}^{n}$. Furthermore, each of the following set forms a $\mathcal{Z}$-basis for $U_{\mathcal{Z}}(n)$ :

(1) $\left\{A(\mathbf{0}) 0(\delta, \lambda) \mid A \in \Theta^{ \pm}(n), \delta, \lambda \in \mathbb{N}^{n}, \delta_{i} \in\{0,1\}, \forall i\right\}$;

(2) $\left\{A(\delta, \lambda) \mid A \in \Theta^{ \pm}(n), \delta, \lambda \in \mathbb{N}^{n}, \delta_{i} \in\{0,1\}, \forall i\right\}$.

We end this section by recalling an important triangular relation in $\mathcal{K}_{\mathcal{Z}}(n)$. For $A=\left(a_{s, t}\right) \in \widetilde{\Theta}(n)$ let

$$
\sigma_{i, j}(A)= \begin{cases}\sum_{s \leqslant i ; t \geqslant j} a_{s, t} & \text { if } i<j \\ \sum_{s \geqslant i ; t \leqslant j} a_{s, t} & \text { if } i>j\end{cases}
$$

Following [1], for $A, B \in \widetilde{\Theta}(n)$, define $B \preccurlyeq A$ if and only if $\sigma_{i, j}(B) \leqslant \sigma_{i, j}(A)$ for all $i \neq j$. Put $B \prec A$ if $B \preccurlyeq A$ and $\sigma_{i, j}(B)<\sigma_{i, j}(A)$ for some $i \neq j$.

According to $[1,5.5(\mathrm{c})]$, for $A \in \Theta^{ \pm}(n)$ and $\lambda \in \mathbb{Z}^{n}$ the following triangular relation holds in $\mathcal{K}_{\mathcal{Z}}(n)$ :

$$
E^{\left(A^{+}\right)}[\operatorname{diag}(\lambda)] F^{\left(A^{-}\right)}=[A+\operatorname{diag}(\lambda-\boldsymbol{\sigma}(A))]+f
$$

where $\boldsymbol{\sigma}(A)=\left(\sigma_{1}(A), \ldots, \sigma_{n}(A)\right)$ with $\sigma_{i}(A)=\sum_{j<i}\left(a_{i, j}+a_{j, i}\right)$ and $f$ is a finite $\mathcal{Z}$-linear combination of $[B]$ with $B \in \widetilde{\Theta}(n)$ such that $B \prec A$.

\section{The algebra $\widetilde{\mathrm{u}}_{\kappa}(n)_{h}$}

Let $U_{\kappa}(n)=U_{\mathcal{Z}}(n) \otimes_{\mathcal{Z}} \mathcal{K}$. We shall denote the images of $E_{i}^{(m)}, F_{i}^{(m)}, A(\delta, \lambda)$, etc. in $U_{\mathcal{K}}(n)$ by the same letters. For $h \geqslant 1$ let $\widetilde{\mathrm{u}}_{\kappa}(n)_{h}$ be the $\kappa$-subalgebra of $U_{k}(n)$ generated by the elements $E_{i}^{(m)}, F_{i}^{(m)}, K_{j}^{ \pm 1},\left[\begin{array}{c}K_{j} ; 0 \\ t\end{array}\right]$ for $1 \leqslant i \leqslant n-1$, 
$1 \leqslant j \leqslant n$ and $0 \leqslant m, t<l p^{h-1}$. If $l^{\prime}$ is an odd number, we let

$$
\mathrm{u}_{k}(n)_{h}=\widetilde{\mathrm{u}}_{k}(n)_{h} /\left\langle K_{1}^{l}-1, \ldots, K_{n}^{l}-1\right\rangle .
$$

The algebra $\mathrm{u}_{k}(n)_{h}$ is called Frobenius-Lusztig kernels of $U_{k}(n)$. We will construct several $k$-bases for $\widetilde{\mathrm{u}}_{k}(n)_{h}$ in 3.7 .

We need some preparation before proving 3.7. The following result is due to $[18,3.2]$ (see also $[15,4.1])$.

Lemma 3.1. Let $m=m_{0}+l m_{1}, 0 \leqslant m_{0} \leqslant l-1, m_{1} \in \mathbb{N}$. Then

$$
\left[\begin{array}{c}
m \\
t
\end{array}\right]_{\varepsilon}=\varepsilon^{l\left(t_{1} l-t_{1} m_{0}-t m_{1}\right)}\left[\begin{array}{c}
m_{0} \\
t_{0}
\end{array}\right]_{\varepsilon}\left(\begin{array}{c}
m_{1} \\
t_{1}
\end{array}\right)
$$

for $0 \leqslant t \leqslant m$, where $t=t_{0}+l t_{1}$ with $0 \leqslant t_{0} \leqslant l-1$ and $t_{1} \in \mathbb{N}$.

Lemma 3.2. The following identity hold in $\kappa:\left(\begin{array}{c}m+p^{h-1} \\ s\end{array}\right)=\left(\begin{array}{c}m \\ s\end{array}\right)$ for $m \in \mathbb{Z}$ and $0 \leqslant s<p^{h-1}$.

Proof. We consider the polynomial ring $\mathcal{k}[x, y]$. Since the characteristic of $\kappa$ is $p$ we see that

$$
\sum_{0 \leqslant j \leqslant p^{h-1}}\left(\begin{array}{c}
p^{h-1} \\
j
\end{array}\right) x^{j} y^{p^{h-1}-j}=(x+y)^{p^{h-1}}=x^{p^{h-1}}+y^{p^{h-1}} .
$$

It follows that $\left(\begin{array}{c}p^{h-1} \\ j\end{array}\right)=0$ for $0<j<p^{h-1}$. This implies that

$$
\left(\begin{array}{c}
m+p^{h-1} \\
s
\end{array}\right)=\sum_{0 \leqslant j \leqslant s}\left(\begin{array}{c}
p^{h-1} \\
j
\end{array}\right)\left(\begin{array}{c}
m \\
s-j
\end{array}\right)=\left(\begin{array}{c}
m \\
s
\end{array}\right)
$$

for $m \in \mathbb{Z}$ and $0 \leqslant s<p^{h-1}$.

We now generalize 3.2 to the quantum case.

Lemma 3.3. Assume $0 \leqslant a<l p^{h-1}$ and $b \in \mathbb{Z}$. Then we have $\left[\begin{array}{c}b+l p^{h-1} \\ a\end{array}\right]_{\varepsilon}=$ $\varepsilon^{-a l p^{h-1}}\left[\begin{array}{l}b \\ a\end{array}\right]_{\varepsilon}$. In particular, we have $\left[\begin{array}{c}b+l^{\prime} p^{h-1} \\ a\end{array}\right]_{\varepsilon}=\left[\begin{array}{l}b \\ a\end{array}\right]_{\varepsilon}$ 
Proof. We write $a=a_{0}+a_{1} l$ and $b=b_{0}+b_{1} l$ with $0 \leqslant a_{0}, b_{0}<l, a_{1} \in \mathbb{N}$ and $b_{1} \in \mathbb{Z}$. If $b \in \mathbb{N}$, then by 3.1 and 3.2 we conclude that

$$
\begin{aligned}
{\left[\begin{array}{c}
b+l p^{h-1} \\
a
\end{array}\right]_{\varepsilon} } & =\varepsilon^{-a l p^{h-1}} \varepsilon^{l\left(a_{1} l-a_{1} b_{0}-a_{1} b_{1} l-a_{0} b_{1}\right)}\left[\begin{array}{c}
b_{0} \\
a_{0}
\end{array}\right]_{\varepsilon}\left(\begin{array}{c}
b_{1}+p^{h-1} \\
a_{1}
\end{array}\right) \\
& =\varepsilon^{-a l p^{h-1}} \varepsilon^{l\left(a_{1} l-a_{1} b_{0}-a_{1} b_{1} l-a_{0} b_{1}\right)}\left[\begin{array}{c}
b_{0} \\
a_{0}
\end{array}\right]_{\varepsilon}\left(\begin{array}{l}
b_{1} \\
a_{1}
\end{array}\right) \\
& =\varepsilon^{-a l p^{h-1}}\left[\begin{array}{l}
b \\
a
\end{array}\right]_{\varepsilon} .
\end{aligned}
$$

Furthermore if $b+l p^{h-1}<0$, then $-b+a-1-l p^{h-1} \geqslant 0$ and hence

$$
\begin{aligned}
{\left[\begin{array}{c}
b+l p^{h-1} \\
a
\end{array}\right]_{\varepsilon} } & =(-1)^{a}\left[\begin{array}{c}
-b+a-1-l p^{h-1} \\
a
\end{array}\right]_{\varepsilon} \\
& =(-1)^{a} \varepsilon^{a l p^{h-1}}\left[\begin{array}{c}
-b+a-1 \\
a
\end{array}\right]_{\varepsilon}=\varepsilon^{-a l p^{h-1}}\left[\begin{array}{l}
b \\
a
\end{array}\right]_{\varepsilon}
\end{aligned}
$$

Now we assume $-l p^{h-1} \leqslant b<0$. According to 3.1 we have

$$
\left[\begin{array}{c}
b+l p^{h-1} \\
a
\end{array}\right]_{\varepsilon}=\varepsilon^{-a l p^{h-1}} \varepsilon^{l\left(a_{1} l-a_{1} b_{0}-a b_{1}\right)}\left[\begin{array}{l}
b_{0} \\
a_{0}
\end{array}\right]_{\varepsilon}\left(\begin{array}{l}
b_{1} \\
a_{1}
\end{array}\right) .
$$

If $a_{0}-b_{0}-1 \geqslant 0$ then $\left[\begin{array}{c}b_{0} \\ a_{0}\end{array}\right]_{\varepsilon}=(-1)^{a_{0}}\left[\begin{array}{c}a_{0}-b_{0}-1 \\ a_{0}\end{array}\right]_{\varepsilon}=0$ and hence, by 3.1 and (3.3.1), we have

$$
\begin{aligned}
{\left[\begin{array}{l}
b \\
a
\end{array}\right]_{\varepsilon} } & =(-1)^{a}\left[\begin{array}{c}
l\left(a_{1}-b_{1}\right)+\left(a_{0}-b_{0}-1\right) \\
a
\end{array}\right]_{\varepsilon} \\
& =(-1)^{a} \varepsilon^{l\left(a_{1} l-a_{1}\left(a_{0}-b_{0}-1\right)-a\left(a_{1}-b_{1}\right)\right)}\left[\begin{array}{c}
a_{0}-b_{0}-1 \\
a_{0}
\end{array}\right]_{\varepsilon}\left(\begin{array}{c}
a_{1}-b_{1} \\
a_{1}
\end{array}\right) \\
& =0 \\
& =\varepsilon^{a l p^{h-1}}\left[\begin{array}{c}
b+l p^{h-1} \\
a
\end{array}\right]_{\varepsilon} .
\end{aligned}
$$


Now we assume $-l p^{h-1} \leqslant b<0$ and $a_{0}-b_{0}-1<0$. Then $a_{1}-b_{1}-1 \geqslant 0$ and $0 \leqslant l+a_{0}-b_{0}-1<l$. According to 3.1 we have

$$
\begin{aligned}
{\left[\begin{array}{l}
b \\
a
\end{array}\right]_{\varepsilon} } & =(-1)^{a}\left[\begin{array}{c}
-b+a-1 \\
a
\end{array}\right]_{\varepsilon} \\
& =(-1)^{a}\left[\begin{array}{c}
l\left(a_{1}-b_{1}-1\right)+\left(l+a_{0}-b_{0}-1\right) \\
a
\end{array}\right]_{\varepsilon} \\
& =(-1)^{a} \varepsilon^{l\left(-a_{1}\left(a_{0}-b_{0}-1\right)-a\left(a_{1}-b_{1}-1\right)\right)}\left[\begin{array}{c}
l+a_{0}-b_{0}-1 \\
a_{0}
\end{array}\right]_{\varepsilon}\left(\begin{array}{c}
a_{1}-b_{1}-1 \\
a_{1}
\end{array}\right) \\
& =(-1)^{a_{1} l+a_{1}} \varepsilon^{l\left(-a_{1}\left(a_{0}-b_{0}-1\right)-a\left(a_{1}-b_{1}-1\right)\right)}\left[\begin{array}{c}
b_{0}-l \\
a_{0}
\end{array}\right]_{\varepsilon}\left(\begin{array}{c}
b_{1} \\
a_{1}
\end{array}\right) .
\end{aligned}
$$

Since $0 \leqslant a_{0}<l$ and $[m+l]_{\varepsilon}=\varepsilon^{-l}[m]_{\varepsilon}$ we see that $\left[\begin{array}{c}b_{0}-l \\ a_{0}\end{array}\right]=\varepsilon^{a_{0} l}\left[\begin{array}{l}b_{0} \\ a_{0}\end{array}\right]_{\varepsilon}$. This implies that

$$
\left[\begin{array}{l}
b \\
a
\end{array}\right]_{\varepsilon}=(-1)^{a_{1} l+a_{1}} \varepsilon^{l\left(a_{0}-a_{1}\left(a_{0}-b_{0}-1\right)-a\left(a_{1}-b_{1}-1\right)\right)}\left[\begin{array}{c}
b_{0} \\
a_{0}
\end{array}\right]_{\varepsilon}\left(\begin{array}{c}
b_{1} \\
a_{1}
\end{array}\right) .
$$

Furthermore since $\varepsilon^{2 l}=1$ and $\left(a_{1}^{2} l-a_{1}\right)-\left(a_{1} l+a_{1}\right)=-2 a_{1}+l a_{1}\left(a_{1}-1\right)$ is even, we see that

$$
\begin{aligned}
\frac{\varepsilon^{l\left(a_{1} l-a_{1} b_{0}-a b_{1}\right)}}{\varepsilon^{l\left(a_{0}-a_{1}\left(a_{0}-b_{0}-1\right)-a\left(a_{1}-b_{1}-1\right)\right)}} & =\varepsilon^{l\left(-2 a b_{1}-2 a_{1} b_{0}-2 a_{0}+2 a_{0} a_{1}\right)} \varepsilon^{l\left(a_{1}^{2} l-a_{1}\right)} \\
& =\varepsilon^{l\left(a_{1}^{2} l-a_{1}\right)}=\varepsilon^{l\left(a_{1} l+a_{1}\right)}=(-1)^{a_{1}(l+1)} .
\end{aligned}
$$

Thus by (3.3.1) and (3.3.2) we conclude that $\left[\begin{array}{c}b+l p^{h-1} \\ a\end{array}\right]_{\varepsilon}=\varepsilon^{-a l p^{h-1}}\left[\begin{array}{l}b \\ a\end{array}\right]_{\varepsilon}$. The proof is completed.

Corollary 3.4. Assume $0 \leqslant a, b<l p^{h-1}$ and $a+b \geqslant l p^{h-1}$. Then $\left[\begin{array}{c}a+b \\ a\end{array}\right]_{\varepsilon}=0$.

Proof. According to 3.3 we have $\left[\begin{array}{c}a+b \\ a\end{array}\right]_{\varepsilon}=\varepsilon^{-a l p^{h-1}}\left[\begin{array}{c}a+b-l p^{h-1} \\ a\end{array}\right]_{\varepsilon}$. Since $0 \leqslant$ $a+b-l p^{h-1}<a$, we see that $\left[\begin{array}{c}a+b-l p^{h-1} \\ a\end{array}\right]_{\varepsilon}=0$. The assertion follows.

Let $\widetilde{\mathrm{u}}_{\kappa}^{0}(n)_{h}$ be the $k$-subalgebra of $\widetilde{\mathrm{u}}_{k}(n)_{h}$ generated by $K_{j}^{ \pm 1},\left[\begin{array}{c}K_{j} ; 0 \\ t\end{array}\right]$ for $1 \leqslant j \leqslant n$ and $0 \leqslant t<l p^{h-1}$. For $h \geqslant 1$ let

$$
\mathbb{N}_{l p^{h-1}}^{n}=\left\{\lambda \in \mathbb{N}^{n} \mid 0 \leqslant \lambda_{i}<l p^{h-1}, \forall i\right\} .
$$

Lemma 3.5. The set $\mathfrak{M}^{0}=\left\{\prod_{1 \leqslant i \leqslant n} K_{i}^{\delta_{i}}\left[\begin{array}{c}K_{i} ; 0 \\ \lambda_{i}\end{array}\right] \mid \delta \in \mathbb{N}^{n}, \delta_{i} \in\{0,1\}, \lambda \in\right.$ $\left.\mathbb{N}_{l p^{h-1}}^{n}\right\}$ forms a $\mathrm{k}$-basis for $\widetilde{\mathbf{u}}_{k}^{0}(n)_{h}$. 
Proof. Let $V_{1}=\operatorname{span}_{\kappa} \mathfrak{M}^{0}$. From 2.1, we see that the set $\mathfrak{M}^{0}$ is linearly independent. Thus it is enough to prove that $\widetilde{\mathrm{u}}_{k}^{0}(n)_{h}=V_{1}$. Let $V_{2}$ be the K-submodule of $\widetilde{\mathrm{u}}_{\kappa}^{0}(n)_{h}$ spanned by the elements $\prod_{1 \leqslant i \leqslant n} K_{i}^{\delta_{i}}\left[\begin{array}{c}K_{i} ; 0 \\ \lambda_{i}\end{array}\right]\left(\delta \in \mathbb{Z}^{n}\right.$, $\lambda \in \mathbb{N}^{n}, 0 \leqslant \lambda_{i}<l p^{h-1}$, for all $\left.i\right)$. According to [19, 2.3(g8)], for $0 \leqslant t, t^{\prime}<$ $l p^{h-1}$ we have

$$
\begin{aligned}
\varepsilon^{t^{\prime} t}\left[\begin{array}{c}
K_{i} ; 0 \\
t^{\prime}
\end{array}\right]\left[\begin{array}{c}
K_{i} ; 0 \\
t
\end{array}\right]= & {\left[\begin{array}{c}
t+t^{\prime} \\
t
\end{array}\right]_{\varepsilon}\left[\begin{array}{c}
K_{i} ; 0 \\
t+t^{\prime}
\end{array}\right] } \\
& -\sum_{0<j \leqslant t^{\prime}}(-1)^{j} \varepsilon^{t\left(t^{\prime}-j\right)}\left[\begin{array}{c}
t+j-1 \\
j
\end{array}\right]_{\varepsilon} K_{i}^{j}\left[\begin{array}{c}
K_{i} ; 0 \\
t^{\prime}-j
\end{array}\right]\left[\begin{array}{c}
K_{i} ; 0 \\
t
\end{array}\right] .
\end{aligned}
$$

Note that by 3.4 we have $\left[\begin{array}{c}t+t^{\prime} \\ t\end{array}\right]_{\varepsilon}\left[\begin{array}{l}K_{i} ; 0 \\ t+t^{\prime}\end{array}\right]=0$ for $0 \leqslant t, t^{\prime}<l p^{h-1}$ with $t+t^{\prime} \geqslant$ $l p^{h-1}$. Thus, by induction on $t^{\prime}$ we see that $\left[\begin{array}{c}K_{i} ; 0 \\ t^{\prime}\end{array}\right]\left[\begin{array}{c}K_{i} ; 0 \\ t\end{array}\right] \in V_{2}$ for $0 \leqslant t, t^{\prime}<$ $l p^{h-1}$. It follows that $\widetilde{\mathrm{u}}_{k}^{0}(n)_{h}=V_{2}$. Furthermore, by the proof of $[19,2.14]$, for $m \geqslant 0$ and $0 \leqslant t<l p^{h-1}$ we have

$$
\begin{aligned}
K_{i}^{m+2}\left[\begin{array}{c}
K_{i} ; 0 \\
t
\end{array}\right] & =\varepsilon^{t}\left(\varepsilon^{t+1}-\varepsilon^{-t-1}\right) K_{i}^{m+1}\left[\begin{array}{c}
K_{i} ; 0 \\
t+1
\end{array}\right]+\varepsilon^{2 t} K_{i}^{m}\left[\begin{array}{c}
K_{i} ; 0 \\
t
\end{array}\right], \\
K_{i}^{-m-1}\left[\begin{array}{c}
K_{i} ; 0 \\
t
\end{array}\right] & =-\varepsilon^{-t}\left(\varepsilon^{t+1}-\varepsilon^{-t-1}\right) K_{i}^{-m}\left[\begin{array}{c}
K_{i} ; 0 \\
t+1
\end{array}\right]+\varepsilon^{-2 t} K_{i}^{-m+1}\left[\begin{array}{c}
K_{i} ; 0 \\
t
\end{array}\right] .
\end{aligned}
$$

If $t+1=l p^{h-1}$, then

$$
\varepsilon^{t}\left(\varepsilon^{t+1}-\varepsilon^{-t-1}\right) K_{i}^{m+1}\left[\begin{array}{c}
K_{i} ; 0 \\
t+1
\end{array}\right]=-\varepsilon^{-t}\left(\varepsilon^{t+1}-\varepsilon^{-t-1}\right) K_{i}^{-m}\left[\begin{array}{c}
K_{i} ; 0 \\
t+1
\end{array}\right]=0
$$

Thus by induction on $m \geqslant 0$ we see that $K_{i}^{ \pm m}\left[\begin{array}{c}K_{i} ; 0 \\ t\end{array}\right] \in V_{1}$ for $0 \leqslant t<l p^{h-1}$. This implies that $V_{1}=V_{2}$. The assertion follows.

We are now ready to prove 3.7. Let $\Theta^{ \pm}(n)_{h}=\left\{A \in \Theta^{ \pm}(n) \mid 0 \leqslant a_{s, t}<\right.$ $\left.l p^{h-1}, \forall s \neq t\right\}$.

Lemma 3.6. The algebra $\widetilde{\mathrm{u}}_{k}(n)_{h}$ is generated as a $\mathcal{K}$-module by the elements $A(\delta, \lambda)$ for $A \in \Theta^{ \pm}(n)_{h}, \delta \in \mathbb{Z}^{n}$ and $\lambda \in \mathbb{N}_{l p^{h-1}}^{n}$.

Proof. Let $V_{h}$ be the $\mathcal{K}$-submodule of $U_{k}(n)$ spanned by $A(\delta, \lambda)$ for $A \in$ $\Theta^{ \pm}(n)_{h}, \delta \in \mathbb{Z}^{n}$ and $\lambda \in \mathbb{N}_{l p^{h-1}}^{n}$. According to $[16,3.5(1)]$ for $A \in \Theta^{ \pm}(n)_{h}$, 
$0 \leqslant m<l p^{h-1}, 1 \leqslant i \leqslant n-1, \delta \in \mathbb{Z}^{n}$ and $\lambda \in \mathbb{N}_{l p^{h-1}}^{n}$, we have

$$
\begin{aligned}
& \left(m E_{i, i+1}\right)(\mathbf{0}) A(\delta, \lambda) \\
& =\sum_{\substack{\mathbf{t} \in \Lambda(n, m), 0 \leqslant j \leqslant \lambda_{i} \\
t_{u} \leqslant a_{i+1, u}, \forall u \neq i+1}} f_{j, c, k}^{\mathbf{t}}\left(A+\sum_{u \neq i} t_{u} E_{i, u}-\sum_{\substack{u \neq i+1 \\
0 \leqslant k \leqslant \lambda_{u}}} t_{u} E_{i+1, u}\right)\left(\delta+\alpha_{j, c, k}^{\mathbf{t}}, \lambda+\beta_{j, c, k}^{\mathbf{t}}\right) . \\
& 0 \leqslant k \leqslant \lambda_{i+1} \\
& 0 \leqslant c \leqslant \min \left\{t_{i}, j\right\}
\end{aligned}
$$

where $\alpha_{j, c, k}^{\mathbf{t}}=\left(\sum_{i>u} t_{u}+\lambda_{i}-j-c\right) \boldsymbol{e}_{i}+\left(\lambda_{i+1}-k-\sum_{i+1>u} t_{u}\right) \boldsymbol{e}_{i+1}, \quad \beta_{j, c, k}^{\mathbf{t}}=$ $\left(t_{i}+j-c-\lambda_{i}\right) \boldsymbol{e}_{i}+\left(k-\lambda_{i+1}\right) \boldsymbol{e}_{i+1}$ with $\boldsymbol{e}_{i}=\left(0, \ldots, 0,{ }_{i}, 0, \ldots, 0\right) \in \mathbb{N}^{n}$, and

$$
f_{j, c, k}^{\mathbf{t}}=\varepsilon^{g_{j, k}^{\mathbf{t}}} \prod_{u \neq i}\left[\begin{array}{c}
a_{i, u}+t_{u} \\
t_{u}
\end{array}\right]_{\varepsilon}\left[\begin{array}{c}
-t_{i} \\
\lambda_{i}-j
\end{array}\right]_{\varepsilon}\left[\begin{array}{c}
t_{i}+j-c \\
t_{i}
\end{array}\right]_{\varepsilon}\left[\begin{array}{c}
t_{i} \\
c
\end{array}\right]_{\varepsilon}\left[\begin{array}{c}
t_{i+1} \\
\lambda_{i+1}-k
\end{array}\right]_{\varepsilon}
$$

with $g_{j, k}^{\mathbf{t}}=\sum_{j>u, j \neq i} a_{i, j} t_{u}-\sum_{j>u, j \neq i+1} a_{i+1, j} t_{u}+\sum_{u^{\prime} \neq i, i+1, u<u^{\prime}} t_{u} t_{u^{\prime}}-t_{i} \delta_{i}+$ $t_{i+1} \delta_{i+1}+2 j t_{i}-k t_{i+1}$. If $A+\sum_{u \neq i} t_{u} E_{i, u}-\sum_{u \neq i+1} t_{u} E_{i+1, u} \notin \Theta^{ \pm}(n)_{h}$ then $a_{i, u}+t_{u} \geqslant l p^{h-1}$ for some $u \neq i$. From 3.4 we see that $\left[\begin{array}{c}a_{i, u}+t_{u} \\ t_{u}\end{array}\right]_{\varepsilon}=0$ and hence $f_{j, c, k}^{\mathbf{t}}=0$. Furthermore, if $\lambda+\beta_{j, c, k}^{\mathbf{t}} \notin \mathbb{N}_{l p^{h-1}}^{n}$ then $\left(\lambda+\beta_{j, c, k}^{t}\right)_{i}=t_{i}+$ $j-c \geqslant l p^{h-1}$. From 3.4 we see that $\left[\begin{array}{c}t_{i}+j-c \\ t_{i}\end{array}\right]_{\varepsilon}=0$ and hence $f_{j, c, k}^{\mathbf{t}}=0$. Thus we conclude that

$$
\left(m E_{i, i+1}\right)(\mathbf{0}) V_{h} \subseteq V_{h},
$$

for $0 \leqslant m<l p^{h-1}$ and $1 \leqslant i \leqslant n-1$. Similarly, using [16, 3.4,3.5(2)] we see that

$$
\left(m E_{i+1, i}\right)(\mathbf{0}) V_{h} \subseteq V_{h} \quad \text { and } \quad 0(\gamma, \mu) V_{h} \subseteq V_{h}
$$

for $0 \leqslant m<l p^{h-1}, 1 \leqslant i \leqslant n-1, \gamma \in \mathbb{Z}^{n}$ and $\mu \in \mathbb{N}_{l p^{h-1}}^{n}$. Combining (3.6.1) with (3.6.2) implies that

$$
\widetilde{\mathrm{u}}_{k}(n)_{h} \subseteq \widetilde{\mathrm{u}}_{\kappa}(n)_{h} V_{h} \subseteq V_{h}
$$

On the other hand, from $[16,3.4]$ we see that for $A \in \Theta^{ \pm}(n)_{h}, \delta \in \mathbb{Z}^{n}$ and $\lambda \in \mathbb{N}_{l p^{h-1}}^{n}$,

$$
\begin{aligned}
A(\mathbf{0}) 0(\delta, \lambda)= & \varepsilon^{\operatorname{co}(A) \cdot(\delta+\lambda)} A(\delta, \lambda) \\
& +\sum_{\mathbf{j} \in \mathbb{N}^{n}, \mathbf{0}<\mathbf{j} \leqslant \lambda} \varepsilon^{\operatorname{co}(A) \cdot(\delta+\lambda-\mathbf{j})}\left[\begin{array}{c}
\operatorname{co}(A) \\
\mathbf{j}
\end{array}\right] A(\delta-\mathbf{j}, \lambda-\mathbf{j}) .
\end{aligned}
$$


This implies that

$$
V_{h}=\operatorname{span}_{k}\left\{A(\mathbf{0}) 0(\delta, \lambda) \mid A \in \Theta^{ \pm}(n)_{h}, \delta \in \mathbb{Z}^{n}, \lambda \in \mathbb{N}_{l p^{h-1}}^{n}\right\} .
$$

Furthermore, combining (2.4.1) with 2.4 shows that for $A \in \Theta^{ \pm}(n)_{h}, \delta \in \mathbb{Z}^{n}$ and $\lambda \in \mathbb{N}_{l p^{h-1}}^{n}$,

$$
E^{\left(A^{+}\right)} F^{\left(A^{-}\right)} \prod_{1 \leqslant i \leqslant n} K_{i}^{\delta_{i}}\left[\begin{array}{c}
K_{i} ; 0 \\
\lambda_{i}
\end{array}\right]=E^{\left(A^{+}\right)} F^{\left(A^{-}\right)} 0(\delta, \lambda)=A(\mathbf{0}) 0(\delta, \lambda)+f
$$

where $f$ is a $\mathcal{k}$-linear combination of $B(\mathbf{0}) 0(\gamma, \mu)$ with $B \in \Theta^{ \pm}(n), B \prec A$, $\gamma \in \mathbb{Z}^{n}$ and $\mu \in \mathbb{N}^{n}$. From (3.6.3) and (3.6.5) we see that $f$ must be a $\mathcal{K}$ linear combination of $B(\mathbf{0}) 0(\gamma, \mu)$ with $B \in \Theta^{ \pm}(n)_{h}, B \prec A, \gamma \in \mathbb{Z}^{n}$ and $\mu \in \mathbb{N}_{l p^{h-1}}^{n}$. Thus we conclude that

$$
\begin{array}{r}
V_{h}=\operatorname{span}_{\kappa}\left\{E^{\left(A^{+}\right)} F^{\left(A^{-}\right)} \prod_{1 \leqslant i \leqslant n} K_{i}^{\delta_{i}}\left[\begin{array}{c}
K_{i} ; 0 \\
\lambda_{i}
\end{array}\right] \mid\right. \\
\left.A \in \Theta^{ \pm}(n)_{h}, \delta \in \mathbb{Z}^{n}, \lambda \in \mathbb{N}_{l p^{h-1}}^{n}\right\} \subseteq \widetilde{\mathrm{u}}_{\kappa}(n)_{h} .
\end{array}
$$

The assertion follows.

Proposition 3.7. Each of the following set forms a $\mathcal{K}$-basis for $\widetilde{\mathrm{u}}_{k}(n)_{h}$ :

(1) $\mathfrak{M}:=\left\{E^{\left(A^{+}\right)} \prod_{1 \leqslant i \leqslant n} K_{i}^{\delta_{i}}\left[\begin{array}{c}K_{i} ; 0 \\ \lambda_{i}\end{array}\right] F^{\left(A^{-}\right)} \mid A \in \Theta^{ \pm}(n)_{h}, \delta \in \mathbb{N}^{n}, \delta_{i} \in\{0,1\}\right.$, $\left.\forall i, \lambda \in \mathbb{N}_{l p^{h-1}}^{n}\right\}$;

(2) $\mathfrak{B}:=\left\{A(\delta, \lambda) \mid A \in \Theta^{ \pm}(n)_{h}, \delta \in \mathbb{N}^{n}, \delta_{i} \in\{0,1\}, \forall i, \lambda \in \mathbb{N}_{l p^{h-1}}^{n}\right\}$;

(3) $\mathfrak{B}^{\prime}:=\left\{A(\mathbf{0}) 0(\delta, \lambda) \mid A \in \Theta^{ \pm}(n)_{h}, \delta \in \mathbb{N}^{n}, \delta_{i} \in\{0,1\}, \forall i, \lambda \in \mathbb{N}_{l p^{h-1}}^{n}\right\}$.

Proof. According to 2.1 and 2.4, it is enough to prove that $\widetilde{\mathrm{u}}_{\mathcal{K}}(n)_{h}=\operatorname{span}_{\mathcal{K}} \mathfrak{M}$ $=\operatorname{span}_{\mathcal{K}} \mathfrak{B}=\operatorname{span}_{\mathcal{K}} \mathfrak{B}^{\prime}$. From 3.5, 3.6, (3.6.5) and (3.6.6) we see that $\widetilde{\mathrm{u}}_{\kappa}(n)_{h}=$ $\operatorname{span}_{\kappa} \mathfrak{M}=\operatorname{span}_{\kappa} \mathfrak{B}^{\prime}$. For $A \in \Theta^{ \pm}(n)_{h}, \delta \in \mathbb{Z}^{n}$ and $\lambda \in \mathbb{N}_{l p^{h-1}}^{n}$ we have

$$
\begin{aligned}
A(\delta, \lambda) & =\varepsilon^{\lambda_{i}}\left(\varepsilon^{\lambda_{i}+1}-\varepsilon^{-\lambda_{i}-1}\right) A\left(\delta-\boldsymbol{e}_{i}, \lambda+\boldsymbol{e}_{i}\right)+\varepsilon^{2 \lambda_{i}} A\left(\delta-2 \boldsymbol{e}_{i}, \lambda\right) \\
& =-\varepsilon^{-\lambda_{i}}\left(\varepsilon^{\lambda_{i}+1}-\varepsilon^{-\lambda_{i}-1}\right) A\left(\delta+\boldsymbol{e}_{i}, \lambda+\boldsymbol{e}_{i}\right)+\varepsilon^{-2 \lambda_{i}} A\left(\delta+2 \boldsymbol{e}_{i}, \lambda\right)
\end{aligned}
$$

Note that if $\lambda_{i}+1=l p^{h-1}$ then $\varepsilon^{\lambda_{i}}\left(\varepsilon^{\lambda_{i}+1}-\varepsilon^{-\lambda_{i}-1}\right) A\left(\delta-\boldsymbol{e}_{i}, \lambda+\boldsymbol{e}_{i}\right)=$ $-\varepsilon^{-\lambda_{i}}\left(\varepsilon^{\lambda_{i}+1}-\varepsilon^{-\lambda_{i}-1}\right) A\left(\delta+\boldsymbol{e}_{i}, \lambda+\boldsymbol{e}_{i}\right)=0$. This together with 3.6 shows that $\widetilde{\mathrm{u}}_{\kappa}(n)_{h}=\operatorname{span}_{\mathcal{K}} \mathfrak{B}$. 


\section{The algebra $\mathscr{K}^{\prime}(n)_{h}$}

We will construct the algebra $\mathscr{K}^{\prime}(n)_{h}$ in this section. We will prove in 5.5 the algebra $\mathscr{K}^{\prime}(n)_{h}$ is the realization of $\widetilde{\mathrm{u}}_{\kappa}(n)_{h}$.

Let $\mathcal{K}_{\mathcal{K}}(n)=\mathcal{K}_{\mathcal{Z}}(n) \otimes_{\mathcal{Z}} \mathcal{K}$, where $\mathcal{K}$ is regarded as a $\mathcal{Z}$-module by specializing $v$ to $\varepsilon$. For $A \in \widetilde{\Theta}(n)$ let

$$
[A]_{\varepsilon}=[A] \otimes 1 \in \mathcal{K}_{\kappa}(n) .
$$

Let $\widetilde{\Theta}(n)_{h}$ be the set of all $A=\left(a_{i, j}\right) \in \widetilde{\Theta}(n)$ such that $a_{i, j}<l p^{h-1}$ for all $i \neq j$. We will denote by $\mathscr{K}(n)_{h}$ the $\mathcal{K}$-submodule of $\mathcal{K}_{\mathcal{K}}(n)$ spanned by the elements $[A]_{\varepsilon}$ with $A \in \widetilde{\Theta}(n)_{h}$.

To construct the algebra $\mathscr{K}^{\prime}(n)_{h}$ we need the following lemma (cf. [1, $6.2]$ and $[14,5.1])$.

Lemma 4.1. (1) $\mathscr{K}(n)_{h}$ is a subalgebra of $\mathcal{K}_{\mathcal{K}}(n)$. It is generated by $\left[m E_{i, i+1}+\operatorname{diag}(\lambda)\right]_{\varepsilon}$ and $\left[m E_{i+1, i}+\operatorname{diag}(\lambda)\right]_{\varepsilon}$ for $0 \leqslant m<l p^{h-1}, 1 \leqslant i \leqslant$ $n-1$ and $\lambda \in \mathbb{Z}^{n}$.

(2) Let $D$ be any diagonal matrix in $\widetilde{\Theta}(n)$. The map $\tau_{D}: \mathscr{K}(n)_{h} \rightarrow$ $\mathscr{K}(n)_{h}$ given by $[A]_{\varepsilon} \rightarrow\left[A+l^{\prime} p^{h-1} D\right]_{\varepsilon}$ is an algebra homomorphism.

Proof. Let $A=\left(a_{s, t}\right) \in \widetilde{\Theta}(n)_{h}$ and $0 \leqslant m<l p^{h-1}$. Assume that $B=\left(b_{s, t}\right) \in$ $\widetilde{\Theta}(n)_{h}$ is such that $B-m E_{i, i+1}$ is a diagonal matrix such that $\operatorname{co}(B)=$ $\operatorname{ro}(A)$. By 2.2 we have

$$
\begin{aligned}
{[B]_{\varepsilon} \cdot[A]_{\varepsilon}=} & \sum_{\substack{\mathbf{t} \in \Lambda(n, m) \\
\forall u \neq i+1, t_{u} \leqslant a_{i+1, u}}} \varepsilon^{\beta(\mathbf{t}, A)} \\
& \times \prod_{1 \leqslant u \leqslant n}\left[\begin{array}{c}
a_{i, u}+t_{u} \\
t_{u}
\end{array}\right]_{\varepsilon}\left[A+\sum_{1 \leqslant u \leqslant n} t_{u}\left(E_{i, u}-E_{i+1, u}\right)\right]_{\varepsilon}
\end{aligned}
$$

where $\beta(\mathbf{t}, A)=\sum_{j>u}\left(a_{i, j}-a_{i+1, j}\right) t_{u}+\sum_{u<u^{\prime}} t_{u} t_{u^{\prime}}$. Assume that $A+$ $\sum_{u} t_{u}\left(E_{i, u}-E_{i+1, u}\right) \notin \widetilde{\Theta}(n)_{h}$ for some $\mathbf{t}$; then $a_{i, u}+t_{u} \geqslant l p^{h-1}$ for some $u \neq i$. Since $0 \leqslant a_{i, u}, t_{u}<l p^{h-1}$, by 3.4 , we conclude that $\left[\begin{array}{c}a_{i, u}+t_{u} \\ t_{u}\end{array}\right]_{\varepsilon}=0$ and hence $[B]_{\varepsilon} \cdot[A]_{\varepsilon} \in \mathscr{K}(n)_{h}$. Similarly, we have $[C]_{\varepsilon} \cdot[A]_{\varepsilon} \in \mathscr{K}^{(n}(n)_{h}$, where $C$ is such that $C-m E_{i+1, i}$ is a diagonal matrix such that $\operatorname{co}(C)=\operatorname{ro}(A)$. Now using $[1,4.6(\mathrm{c})],(1)$ can be proved in a way similar to the proof of $[1,6.2]$.

By 2.2 and 3.3 we conclude that $\tau_{D}\left(\left[A^{\prime}\right]_{\varepsilon}[A]_{\varepsilon}\right)=\tau_{D}\left(\left[A^{\prime}\right]_{\varepsilon}\right) \tau_{D}\left([A]_{\varepsilon}\right)$ for any $A^{\prime}$ of the form $B, C$ as above. Since $\mathscr{K}(n)_{h}$ is generated by elements like $[B]_{\varepsilon},[C]_{\varepsilon}$ above, we conclude that $\tau_{D}$ is an algebra homomorphism. 
Let $\widetilde{\Theta}^{\prime}(n)_{h}$ be the set of all $n \times n$ matrices $A=\left(a_{i, j}\right)$ with $a_{i, j} \in \mathbb{N}, a_{i, j}<$ $l p^{h-1}$ for all $i \neq j$ and $a_{i, i} \in \mathbb{Z} / l^{\prime} p^{h-1} \mathbb{Z}$ for all $i$. We have an obvious map $p r$ : $\widetilde{\Theta}(n)_{h} \rightarrow \widetilde{\Theta}^{\prime}(n)_{h}$ defined by reducing the diagonal entries modulo $l^{\prime} p^{h-1} \mathbb{Z}$.

Let $\mathscr{K}^{\prime}(n)_{h}$ be the free $\mathcal{K}$-module with basis $\left\{[A]_{\varepsilon} \mid A \in \widetilde{\Theta}^{\prime}(n)_{h}\right\}$. We shall define an algebra structure on $\mathscr{K}^{\prime}(n)_{h}$ as follows. If the column sums of $A$ are not equal to the row sums of $A^{\prime}$ (as integers modulo $l^{\prime} p^{h-1}$ ), then the product $[A]_{\varepsilon} \cdot\left[A^{\prime}\right]_{\varepsilon}$ for $A, A^{\prime} \in \widetilde{\Theta}^{\prime}(n)_{h}$ is zero. Assume now that the column sums of $A$ are equal to the row sums of $A^{\prime}$ (as integers modulo $l^{\prime} p^{h-1}$ ). We can then represent $A, A^{\prime}$ by elements $\widetilde{A}, \widetilde{A}^{\prime} \in \widetilde{\Theta}(n)_{h}$ such that the column sums of $\widetilde{A}$ are equal to the row sums of $\widetilde{A}^{\prime}$ (as integers). According to 4.1(1), we can write $[\widetilde{A}]_{\varepsilon} \cdot\left[\widetilde{A}^{\prime}\right]_{\varepsilon}=\sum_{\widetilde{A}^{\prime \prime} \in I} \rho_{\widetilde{A}^{\prime \prime}}\left[\widetilde{A}^{\prime \prime}\right]_{\varepsilon}$ (product in $\left.\mathscr{K}(n)_{h}\right)$ where $I=\left\{\widetilde{A}^{\prime \prime} \in\right.$ $\left.\widetilde{\Theta}(n)_{h} \mid \operatorname{ro}\left(\widetilde{A}^{\prime \prime}\right)=\operatorname{ro}(\widetilde{A}), \operatorname{co}\left(\widetilde{A}^{\prime \prime}\right)=\operatorname{co}\left(\widetilde{A}^{\prime}\right)\right\}$ (a finite set) and $\rho_{\widetilde{A}^{\prime \prime}} \in \mathcal{K}$. Then the product $[A]_{\varepsilon} \cdot\left[A^{\prime}\right]_{\varepsilon}$ is defined to be $\sum_{\widetilde{A}^{\prime \prime} \in I} \rho_{\widetilde{A}^{\prime \prime}}\left[\operatorname{pr}\left(\widetilde{A}^{\prime \prime}\right)\right]_{\varepsilon}$. From $4.1(2)$ we see that the product is well defined and $\mathscr{K}^{\prime}(n)_{h}$ becomes an associative algebra over $k$.

In the case where $l^{\prime}$ is odd, the algebra $\mathscr{K}^{\prime}(n)_{1}$ is the algebra $\mathscr{K}^{\prime}$ constructed in [1, 6.3]. Furthermore, it was remarked at the end of [1] that $\mathscr{K}^{\prime}$ is "essentially" the algebra defined in $[19, \S 5]$ for type $A$. We will prove in 5.5 that $\mathscr{K}^{\prime}(n)_{h}$ is isomorphic to the algebra $\mathrm{u}_{k}(n)_{h}$ in the case where $l^{\prime}$ is odd.

Mimicking the construction of $\widehat{\mathcal{K}}_{\mathcal{Q}}(n)$, we define $\widehat{\mathcal{K}}_{\mathcal{K}}(n)$ to be the $\mathcal{K}$ module of all formal $\mathcal{K}$-linear combinations $\sum_{A \in \widetilde{\Theta}(n)} \beta_{A}[A]_{\varepsilon}$ satisfying the property (2.2.1). The product of two elements $\sum_{A \in \widetilde{\Theta}(n)} \beta_{A}[A]_{\varepsilon}$, $\sum_{B \in \widetilde{\Theta}(n)} \gamma_{B}[B]_{\varepsilon}$ in $\widehat{\mathcal{K}}_{\kappa}(n)$ is defined to be $\sum_{A, B} \beta_{A} \gamma_{B}[A]_{\varepsilon} \cdot[B]_{\varepsilon}$ where $[A]_{\varepsilon}$. $[B]_{\varepsilon}$ is the product in $\mathcal{K}_{\mathcal{K}}(n)$. Then $\widehat{\mathcal{K}}_{\mathcal{K}}(n)$ becomes an associative algebra over $\kappa$.

We end this section by interpreting $\mathscr{K}^{\prime}(n)_{h}$ as a $\mathcal{K}$-subalgebra of $\widehat{\mathcal{K}}_{k}(n)$. For $h \geqslant 1$ let $\mathbb{Z}_{l^{\prime} p^{h-1}}=\mathbb{Z} / l^{\prime} p^{h-1} \mathbb{Z}$ and let ${ }^{-}: \mathbb{Z}^{n} \rightarrow\left(\mathbb{Z}_{l^{\prime} p^{h-1}}\right)^{n}$ be the map defined by $\overline{\left(j_{1}, j_{2}, \ldots, j_{n}\right)}=\left(\overline{j_{1}}, \overline{j_{2}}, \ldots, \overline{j_{n}}\right)$. For $A \in \Theta^{ \pm}(n)_{h}$ and $\bar{\mu} \in\left(\mathbb{Z}_{l^{\prime} p^{h-1}}\right)^{n}$ let

$$
\llbracket A+\operatorname{diag}(\bar{\mu}) \rrbracket_{h}=\sum_{\substack{\nu \in \mathbb{Z}^{n} \\ \bar{\mu}=\bar{\nu}}}[A+\operatorname{diag}(\nu)]_{\varepsilon} .
$$

Let $\mathcal{W}_{k}(n)_{h}$ be the $\mathcal{K}$-submodule of $\widehat{\mathcal{K}}_{k}(n)$ spanned by the set $\{\llbracket A+$ $\left.\operatorname{diag}(\bar{\lambda}) \rrbracket_{h} \mid A \in \Theta^{ \pm}(n)_{h}, \bar{\lambda} \in\left(\mathbb{Z}_{l^{\prime} p^{h-1}}\right)^{n}\right\}$. From 4.1 we see that $\mathcal{W}_{k}(n)_{h}$ is a $\mathcal{K}$-subalgebra of $\widehat{\mathcal{K}}_{\kappa}(n)$. Furthermore, it is easy to prove that there is an algebra isomorphism

$$
\mathcal{W}_{k}(n)_{h} \stackrel{\sim}{\rightarrow} \mathscr{K}^{\prime}(n)_{h}
$$


defined by sending $\llbracket A \rrbracket_{h}$ to $[A]_{\varepsilon}$ for $A \in \widetilde{\Theta}^{\prime}(n)_{h}$.

\section{Realization of $\mathrm{u}_{k}(\boldsymbol{n})_{h}$}

For $A \in \Theta^{ \pm}(n), \delta \in \mathbb{Z}^{n}$ and $\lambda \in \mathbb{N}^{n}$ let

$$
A(\delta, \lambda)_{\varepsilon}=\sum_{\mu \in \mathbb{Z}^{n}} \varepsilon^{\mu \cdot \delta}\left[\begin{array}{l}
\mu \\
\lambda
\end{array}\right]_{\varepsilon}[A+\operatorname{diag}(\mu)]_{\varepsilon} \in \widehat{\mathcal{K}}_{\mathcal{K}}(n) .
$$

Let $\mathcal{V}_{\kappa}(n)$ be the $\mathcal{K}$-submodule of $\widehat{\mathcal{K}}_{\kappa}(n)$ spanned by the elements $A(\delta, \lambda)_{\varepsilon}$ for $A \in \Theta^{ \pm}(n), \delta \in \mathbb{Z}^{n}$ and $\lambda \in \mathbb{N}^{n}$. For $h \geqslant 1$ let $\mathcal{V}_{k}(n)_{h}$ be the $\mathcal{K}$-submodule of $\widehat{\mathcal{K}}_{k}(n)$ spanned by the elements $A(\delta, \lambda)_{\varepsilon}$ for $A \in \Theta^{ \pm}(n)_{h}, \delta \in \mathbb{Z}^{n}$ and $\lambda \in$ $\mathbb{N}_{l p^{h-1}}^{n}$. We will prove in 5.5 that $\mathrm{u}_{k}(n)_{h} \cong \mathcal{V}_{k}(n)_{h} \cong \mathscr{K}^{\prime}(n)_{h}$ in the case where $l^{\prime}$ is odd, and that $\widetilde{\mathrm{u}}_{\mathcal{K}}(n)_{h} \cong \mathcal{V}_{k}(n)_{h} \cong \mathscr{K}^{\prime}(n)_{h}$ in the case where $l^{\prime}$ is even and $k$ is a field.

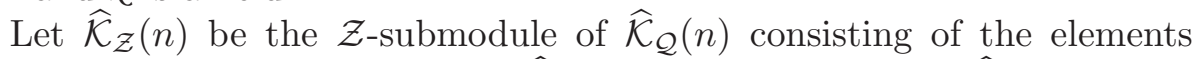
$\sum_{A \in \widetilde{\Theta}(n)} \beta_{A}[A]$ with $\beta_{A} \in \mathcal{Z}$. Then $\widehat{\mathcal{K}}_{\mathcal{Z}}(n)$ is a $\mathcal{Z}$-subalgebra of $\widehat{\mathcal{K}}_{\mathcal{Q}}(n)$. There is a natural algebra homomorphism

$$
\theta: \widehat{\mathcal{K}}_{\mathcal{Z}}(n) \otimes_{\mathcal{Z}} \mathcal{K} \rightarrow \widehat{\mathcal{K}}_{k}(n)
$$

defined by sending $\left(\sum_{A \in \widetilde{\Theta}(n)} \beta_{A}[A]\right) \otimes 1$ to $\sum_{A \in \widetilde{\Theta}(n)}\left(\beta_{A} \cdot 1\right)[A]_{\varepsilon}$, where 1 is the identity element in $\mathcal{K}$.

Recall the injective algebra homomorphism $\varphi: U_{\mathcal{Q}}(n) \rightarrow \widehat{\mathcal{K}}_{\mathcal{Q}}(n)$ defined in 2.3. From 2.4 we see that $\varphi\left(U_{\mathcal{Z}}(n)\right) \subseteq \widehat{\mathcal{K}}_{\mathcal{Z}}(n)$. Thus, by restriction, we get a map $\varphi: U_{\mathcal{Z}}(n) \rightarrow \widehat{\mathcal{K}}_{\mathcal{Z}}(n)$. It induces an algebra homomorphism $\varphi_{\mathcal{K}}$ : $U_{\mathcal{K}}(n) \rightarrow \widehat{\mathcal{K}}_{\mathcal{Z}}(n) \otimes_{\mathcal{Z}} \mathcal{K}$. The map $\theta$, composed with $\varphi_{\mathcal{K}}$ gives an algebra homomorphism

$$
\xi:=\theta \circ \varphi_{\kappa}: U_{\kappa}(n) \rightarrow \widehat{\mathcal{K}}_{\kappa}(n)
$$

By definition we have $\xi(A(\delta, \lambda))=A(\delta, \lambda)_{\varepsilon}$ for $A \in \Theta^{ \pm}(n), \delta \in \mathbb{Z}^{n}$ and $\lambda \in \mathbb{N}^{n}$. This together with 2.4 and 3.6 implies that

$$
\xi\left(U_{k}(n)\right)=\mathcal{V}_{k}(n) \text { and } \xi\left(\widetilde{\mathrm{u}}_{\kappa}(n)_{h}\right)=\mathcal{V}_{k}(n)_{h}
$$

In particular, $\mathcal{V}_{\mathcal{k}}(n)$ and $\mathcal{V}_{\mathcal{K}}(n)_{h}$ are all $\mathcal{K}$-subalgebras of $\widehat{\mathcal{K}}_{\mathcal{k}}(n)$.

We will now construct several bases for $\mathcal{V}_{k}(n)_{h}$ and $\mathcal{V}_{k}(n)$ in 5.1 and 5.3. These results will be used to prove 5.5. According to 3.3 we see that $\left[\begin{array}{l}\nu \\ \lambda\end{array}\right]_{\varepsilon}=$ 
$\left[\begin{array}{c}\nu+l^{\prime} p^{h-1} \delta \\ \lambda\end{array}\right]_{\varepsilon}$ for $\lambda \in \mathbb{N}_{l p^{h-1}}^{n}$ and $\nu, \delta \in \mathbb{Z}^{n}$. This implies that

$$
A(\delta, \lambda)_{\varepsilon}=\sum_{\bar{\mu} \in\left(\mathbb{Z}_{l^{\prime} p^{h}-1}\right)^{n}} \varepsilon^{\delta \cdot \mu}\left[\begin{array}{l}
\mu \\
\lambda
\end{array}\right]_{\varepsilon} \llbracket A+\operatorname{diag}(\bar{\mu}) \rrbracket_{h}
$$

for $A \in \Theta^{ \pm}(n)_{h}, \delta \in \mathbb{Z}^{n}$ and $\lambda \in \mathbb{N}_{l p^{h-1}}^{n}$, where $\llbracket A+\operatorname{diag}(\bar{\mu}) \rrbracket_{h}$ is defined in (4.1.1). For $\lambda, \mu \in \mathbb{N}^{n}$, we write $\lambda \leqslant \mu$ if and only if $\lambda_{i} \leqslant \mu_{i}$ for $1 \leqslant i \leqslant n$. If $\lambda \leqslant \mu$ and $\lambda_{i}<\mu_{i}$ for some $1 \leqslant i \leqslant n$ then we write $\lambda<\mu$.

Lemma 5.1. Assume $l^{\prime}$ is odd. Then $\mathcal{V}_{k}(n)_{h}=\mathcal{W}_{k}(n)_{h}$ and the set $\mathcal{N}_{h}:=$ $\left\{A(\mathbf{0}, \lambda)_{\varepsilon} \mid A \in \Theta^{ \pm}(n)_{h}, \lambda \in \mathbb{N}_{l p^{h-1}}^{n}\right\}$ forms a $\mathcal{k}$-basis for $\mathcal{V}_{k}(n)_{h}$. Furthermore, if $p>0$, then the set $\mathcal{N}:=\left\{A(\mathbf{0}, \lambda) \mid A \in \Theta^{ \pm}(n), \lambda \in \mathbb{N}^{n}\right\}$ forms a $\kappa$-basis for $\mathcal{V}_{k}(n)$.

Proof. From (5.0.3) we see that for $A \in \Theta^{ \pm}(n)_{h}$ and $\lambda \in \mathbb{N}_{l p^{h-1}}^{n}$,

$$
A(\mathbf{0}, \lambda)_{\varepsilon}=\llbracket A+\operatorname{diag}(\bar{\lambda}) \rrbracket_{h}+\sum_{\mu \in \mathbb{N}_{l p}^{n} l^{h-1}, \lambda<\mu}\left[\begin{array}{c}
\mu \\
\lambda
\end{array}\right]_{\varepsilon} \llbracket A+\operatorname{diag}(\bar{\mu}) \rrbracket_{h} .
$$

This, together with the fact that the set $\left\{\llbracket A+\operatorname{diag}(\bar{\lambda}) \rrbracket_{h} \mid A \in \Theta^{ \pm}(n)_{h}, \bar{\lambda} \in\right.$ $\left.\left(\mathbb{Z}_{l p^{h-1}}\right)^{n}\right\}$ forms a $\mathcal{K}$-basis for $\mathcal{W}_{\mathcal{K}}(n)_{h}$, shows that the set $\mathcal{N}_{h}$ forms a $\mathcal{K}$-basis for $\mathcal{W}_{k}(n)_{h}$. It follows that $\mathcal{W}_{k}(n)_{h} \subseteq \mathcal{V}_{k}(n)_{h}$. Furthermore from (5.0.3) we see that $\mathcal{V}_{k}(n)_{h} \subseteq \mathcal{W}_{k}(n)_{h}$. Thus $\mathcal{V}_{k}(n)_{h}=\mathcal{W}_{k}(n)_{h}$. Now we assume $p=$ chark $>0$. Since $\mathcal{V}_{\mathcal{k}}(n)=\bigcup_{h \geqslant 1} \mathcal{V}_{k}(n)_{h}, \mathcal{N}=\bigcup_{h \geqslant 1} \mathcal{N}_{h}$ and the set $\mathcal{N}_{h}$ forms a $\mathcal{K}$-basis for $\mathcal{V}_{\mathcal{K}}(n)_{h}$, we conclude that the set $\mathcal{N}$ forms a $\mathcal{K}$-basis for $\mathcal{V}_{k}(n)$.

Lemma 5.2. For $m \geqslant 1$, let $X_{m}=\left((-1)^{\delta \cdot \beta}\right)_{\delta, \beta \in \mathcal{I}_{m}}$, where $\mathcal{I}_{m}=\left\{\delta \in \mathbb{N}^{m} \mid\right.$ $\delta_{i} \in\{0,1\}$ for $\left.1 \leqslant i \leqslant m\right\}$. If we order the set $\mathcal{I}_{m}$ lexicographically, then $\operatorname{det}\left(X_{m}\right)=(-2)^{2^{m-1} m}$ for all $m$.

Proof. Since $\mathcal{I}_{m}=\left\{(0, \delta) \mid \delta \in \mathcal{I}_{m-1}\right\} \cup\left\{(1, \delta) \mid \delta \in \mathcal{I}_{m-1}\right\}$ we see that

$$
X_{m}=\left(\begin{array}{cc}
X_{m-1} & X_{m-1} \\
X_{m-1} & -X_{m-1}
\end{array}\right) .
$$

This, together with the fact that $\operatorname{det}\left(X_{1}\right)=-2$, implies that

$$
\operatorname{det}\left(X_{m}\right)=\operatorname{det}\left(\begin{array}{cc}
X_{m-1} & X_{m-1} \\
0 & -2 X_{m-1}
\end{array}\right)=(-2)^{2^{m-1}} \operatorname{det}\left(X_{m-1}\right)^{2}=(-2)^{2^{m-1} m}
$$

as required. 
Corollary 5.3. Assume $l^{\prime}$ is even and $\mathcal{K}$ is a field. Then $\mathcal{V}_{k}(n)_{h}=\mathcal{W}_{k}(n)_{h}$ and the set $\mathcal{B}_{h}:=\left\{A(\delta, \lambda)_{\varepsilon} \mid A \in \Theta^{ \pm}(n)_{h}, \lambda \in \mathbb{N}_{l p^{h-1}}^{n}, \delta \in \mathbb{N}^{n}, \delta_{i} \in\{0,1\}, \forall i\right\}$ forms a $\mathcal{K}$-basis for $\mathcal{V}_{\mathcal{K}}(n)_{h}$. Furthermore, if $p>0$, then the set $\mathcal{B}:=\{A(\delta, \lambda) \mid$ $\left.A \in \Theta^{ \pm}(n), \lambda, \delta \in \mathbb{N}^{n}, \delta_{i} \in\{0,1\}, \forall i\right\}$ forms a $\mathcal{K}$-basis for $\mathcal{V}_{k}(n)$.

Proof. Note that there is a bijective map from $\left\{(\delta, \lambda) \mid \delta \in \mathbb{N}^{n}, \delta_{i} \in\{0,1\}\right.$, $\left.\lambda \in \mathbb{N}_{l p^{h-1}}^{n}\right\}$ to $\left(\mathbb{Z}_{l^{\prime} p^{h-1}}\right)^{n}$ defined by sending $(\delta, \lambda)$ to $\overline{\lambda+l p^{h-1} \delta}$. Thus by (5.0.3) and 3.3 we conclude that for $A \in \Theta^{ \pm}(n)_{h}, \lambda \in \mathbb{N}_{l p^{h-1}}^{n}$ and $\delta \in \mathbb{N}^{n}$

$$
\begin{aligned}
& A(\delta, \lambda)_{\varepsilon}=\sum_{\substack{\beta \in \mathbb{N}^{n}, \beta_{i} \in\{0,1\}, \forall i \\
\alpha \in \mathbb{N}^{n} p^{h-1}}} \varepsilon^{\delta .\left(\alpha+l p^{h-1} \beta\right)}\left[\begin{array}{c}
\alpha+l p^{h-1} \beta \\
\lambda
\end{array}\right]_{\varepsilon} \llbracket A+\operatorname{diag}\left(\overline{\alpha+l p^{h-1} \beta}\right) \rrbracket_{h} \\
& =\sum_{\substack{\beta \in \mathbb{N}^{n}, \beta_{i} \in\{0,1\}, \forall i \\
\alpha \in \mathbb{N}_{l p} l_{h-1}}} \varepsilon^{\delta \cdot \alpha} \varepsilon^{l p^{h-1}(\delta \cdot \beta-\beta . \lambda)}\left[\begin{array}{l}
\alpha \\
\lambda
\end{array}\right]_{\varepsilon} \llbracket A+\operatorname{diag}\left(\overline{\alpha+l p^{h-1} \beta}\right) \rrbracket_{h} .
\end{aligned}
$$

Since $l^{\prime}$ is even and $\left(l^{\prime}, p\right)=1$ we see that $p$ is an odd prime. This, together with the fact that $\varepsilon^{l}=-1$, implies that $\varepsilon^{l p^{h-1}}=(-1)^{p^{h-1}}=-1$. Thus for $A \in \Theta^{ \pm}(n)_{h}, \lambda \in \mathbb{N}_{l p^{h-1}}^{n}$ and $\delta \in \mathbb{N}^{n}$ we have

$$
\begin{aligned}
\text { (5.3.1) } A(\delta, \lambda)_{\varepsilon}= & \sum_{\substack{\beta \in \mathbb{N}^{n}, \beta_{i} \in\{0,1\}, \forall i \\
\alpha \in \mathbb{N}_{l p^{h-1}}^{n}}} \varepsilon^{\delta \cdot \alpha}(-1)^{\beta \cdot(\delta-\lambda)}\left[\begin{array}{c}
\alpha \\
\lambda
\end{array}\right]_{\varepsilon} \llbracket A+\operatorname{diag}\left(\overline{\alpha+l p^{h-1} \beta}\right) \rrbracket_{h} \\
= & \sum_{\beta \in \mathbb{N}^{n}, \beta_{i} \in\{0,1\}, \forall i} \varepsilon^{\delta \cdot \lambda}(-1)^{\beta \cdot(\delta-\lambda)} \llbracket A+\operatorname{diag}\left(\overline{\lambda+l p^{h-1} \beta}\right) \rrbracket_{h} \\
& +\sum_{\substack{\beta \in \mathbb{N}^{\mathbb{N}}, \beta_{i} \in\{0,1\}, \forall i \\
\alpha \in \mathbb{N}_{l}^{n} h-1, \lambda<\alpha}} \varepsilon^{\delta \cdot \alpha}(-1)^{\beta \cdot(\delta-\lambda)}\left[\begin{array}{c}
\alpha \\
\lambda
\end{array}\right]_{\varepsilon} \llbracket A+\operatorname{diag}\left(\overline{\alpha+l p^{h-1} \beta}\right) \rrbracket_{h} .
\end{aligned}
$$

From 5.2 we see that for $\lambda \in \mathbb{N}_{l p^{h-1}}^{n}$,

$$
\begin{aligned}
\operatorname{det}\left(\varepsilon^{\delta \cdot \lambda}(-1)^{\beta \cdot(\delta-\lambda)}\right)_{\delta, \beta \in \mathcal{I}_{n}} & =(-\varepsilon)^{\sum_{\delta \in \mathcal{I}_{n}} \lambda \cdot \delta}(-2)^{2^{n-1} n} \\
& =(-\varepsilon)^{\sum_{\delta \in \mathcal{I}_{n}} \lambda \cdot \delta}\left(\varepsilon^{l}-1\right)^{2^{n-1} n} \\
& \neq 0,
\end{aligned}
$$

where $\mathcal{I}_{n}=\left\{\delta \in \mathbb{N}^{n} \mid \delta_{i} \in\{0,1\}\right.$ for $\left.1 \leqslant i \leqslant n\right\}$. It follows that the martix $\left(\varepsilon^{\delta \cdot \lambda}(-1)^{\beta \cdot(\delta-\lambda)}\right)_{\delta, \beta \in \mathcal{I}_{n}}$ is invertible since $\mathcal{K}$ is a field. Thus by (5.3.1) we conclude that the set $\mathcal{B}_{h}$ forms a $\mathcal{k}$-basis for $\mathcal{W}_{k}(n)_{h}$ and $\mathcal{V}_{k}(n)_{h}=\mathcal{W}_{k}(n)_{h}$. Now we assume $p=\operatorname{char} \mathcal{K}>0$. Then $\mathcal{B}=\bigcup_{h \geqslant 1} \mathcal{B}_{h}$. Since the set $\mathcal{B}_{h}$ is linear independent for all $h$, we conclude that the set $\mathcal{B}$ is linear independent. Consequently, the set $\mathcal{B}$ forms a $\mathcal{K}$-basis for $\mathcal{V}_{k}(n)$. 
We are now ready to prove the main result of this paper.

Theorem 5.4. (1) If $l^{\prime}$ is odd, then $\operatorname{ker}(\xi)=\left\langle K_{i}^{l}-1 \mid 1 \leqslant i \leqslant n\right\rangle$ and hence $U_{k}(n) /\left\langle K_{i}^{l}-1 \mid 1 \leqslant i \leqslant n\right\rangle \cong \mathcal{V}_{k}(n)$.

(2) If $l^{\prime}$ is even and $\mathcal{K}$ is a field with $p=\operatorname{char} \mathcal{K}>0$, then $\xi$ is injective and hence $U_{k}(n) \cong \mathcal{V}_{k}(n)$.

Proof. The assertion (1) can be proved in a way similar to the proof of [16, 4.6]. The assertion (2) follows from 2.4, 5.3 and (5.0.2).

Theorem 5.5. (1) If $l^{\prime}$ is odd, then $\mathrm{u}_{k}(n)_{h} \cong \mathcal{V}_{k}(n)_{h} \cong \mathscr{K}^{\prime}(n)_{h}$ for $h \geqslant 1$.

(2) If $l^{\prime}$ is even and $\mathcal{K}$ is a field, then $\widetilde{\mathrm{u}}_{\mathcal{K}}(n)_{h} \cong \mathcal{V}_{\mathcal{K}}(n)_{h} \cong \mathscr{K}^{\prime}(n)_{h}$ for $h \geqslant 1$.

Proof. If either $l^{\prime}$ is odd or both $l^{\prime}$ is even and $\mathcal{K}$ is a field, then by (4.1.2), 5.1 and 5.3, we deduce that $\mathcal{V}_{k}(n)_{h} \cong \mathscr{K}^{\prime}(n)_{h}$. If $l^{\prime}$ is odd, then $\xi\left(K_{i}^{l}-1\right)=0$ and hence the map $\xi: U_{\mathcal{K}}(n) \rightarrow \widehat{\mathcal{K}}_{\mathcal{K}}(n)$ induces an algebra homomorphism

$$
\bar{\xi}: U_{k}(n) /\left\langle K_{i}^{l}-1 \mid 1 \leqslant i \leqslant n\right\rangle \rightarrow \widehat{\mathcal{K}}_{k}(n) .
$$

One can prove that the set $\left\{E^{\left(A^{+}\right)} \prod_{1 \leqslant i \leqslant n} K_{i}^{-\lambda_{i}}\left[\begin{array}{c}K_{i} ; 0 \\ \lambda_{i}\end{array}\right] F^{\left(A^{-}\right)} \mid A \in \Theta^{ \pm}(n)_{h}\right.$, $\left.\lambda \in \mathbb{N}_{l p^{h-1}}^{n}\right\}$ forms a $\mathcal{K}$-basis of $\mathrm{u}_{k}(n)_{h}$ in a way similar to the proof of $[19,6.5]$. Thus we may regard $\mathrm{u}_{k}(n)_{h}$ as a $\mathcal{K}$-subalgebra of $U_{k}(n) /\left\langle K_{i}^{l}-1 \mid 1 \leqslant i \leqslant n\right\rangle$. From (5.0.2) we see that $\bar{\xi}\left(\mathrm{u}_{k}(n)_{h}\right)=\mathcal{V}_{k}(n)_{h}$. Thus the restriction of $\bar{\xi}$ to $\mathrm{u}_{k}(n)_{h}$ yields a surjective algebra homomorphism

$$
\bar{\xi}^{\prime}: \mathrm{u}_{k}(n)_{h} \rightarrow \mathcal{V}_{k}(n)_{h}
$$

This, together with 5.4(1), implies that $\mathrm{u}_{k}(n)_{h} \cong \mathcal{V}_{k}(n)_{h}$. Now we assume $l^{\prime}$ is even and $\mathcal{K}$ is a field. Since $\xi\left(\widetilde{\mathrm{u}}_{\mathcal{K}}(n)_{h}\right)=\mathcal{V}_{\mathcal{k}}(n)_{h}$ by $(5.0 .2)$, the restriction of $\xi$ to $\widetilde{\mathrm{u}}_{\kappa}(n)_{h}$ yields a surjective algebra homomorphism

$$
\xi^{\prime}: \widetilde{\mathrm{u}}_{k}(n)_{h} \rightarrow \mathcal{V}_{k}(n)_{h}
$$

From 3.7 and 5.3 we see that $\xi^{\prime}$ is injective. Consequently, $\widetilde{\mathrm{u}}_{\kappa}(n)_{h} \cong \mathcal{V}_{k}(n)_{h}$.

\section{The infinitesimal $q$-Schur algebras and little $q$-Schur algebras}

Let $\mathcal{S}_{\mathcal{Z}}(n, r)$ be the algebra over $\mathcal{Z}$ introduced in $[1,1.2]$. It has a $\mathcal{Z}$ basis $\{[A] \mid A \in \Theta(n, r)\}$ defined in [1], where $\Theta(n, r)=\{A \in \Theta(n) \mid \sigma(A):=$ 
$\left.\sum_{1 \leqslant i, j \leqslant n} a_{i, j}=r\right\}$. It is proved in [8, A.1] that the algebra $\mathcal{S}_{\mathcal{Z}}(n, r)$ is isomorphic to the $q$-Schur algebra introduced in $[4,5]$. Let $\mathcal{S}_{k}(n, r)=\mathcal{S}_{\mathcal{Z}}(n, r) \otimes \mathcal{Z}$ k. For $A \in \Theta(n, r)$ let

$$
[A]_{\varepsilon}=[A] \otimes 1 \in \mathcal{S}_{\kappa}(n, r) .
$$

Recall that $\Lambda(n, r)=\left\{\lambda \in \mathbb{N}^{n} \mid \sum_{1 \leqslant i \leqslant n} \lambda_{i}=r\right\}$. Let $\overline{\Lambda(n, r)}_{l^{\prime} p^{h-1}}=\{\bar{\lambda} \in$ $\left.\left(\mathbb{Z}_{l^{\prime} p^{h-1}}\right)^{n} \mid \lambda \in \Lambda(n, r)\right\}$. For $A \in \Theta^{ \pm}(n)_{h}$ and $\bar{\lambda} \in\left(\mathbb{Z}_{l^{\prime} p^{h-1}}\right)^{n}$ we define the element $\llbracket A+\operatorname{diag}(\bar{\lambda}), r \rrbracket_{h} \in \mathcal{S}_{k}(n, r)$ as follows:

$$
\llbracket A+\operatorname{diag}(\bar{\lambda}), r \rrbracket_{h}=\sum_{\substack{\mu \in \Lambda(n, r-\sigma) \\ \mu=\bar{\lambda}}}[A+\operatorname{diag}(\mu)]_{\varepsilon}
$$

Note that $\llbracket A+\operatorname{diag}(\bar{\lambda}), r \rrbracket_{h}=0$ if either $\sigma(A)>r$ or $\bar{\lambda} \notin \overline{\Lambda(n, r-\sigma(A))_{l^{\prime}} p^{h-1}}$. Let $\widetilde{\mathrm{u}}_{\kappa}(n, r)_{h}$ be the $\kappa$-submodule of $\mathcal{S}_{\kappa}(n, r)$ spanned by the set $\{\llbracket A+$ $\left.\operatorname{diag}(\bar{\lambda}), r \rrbracket_{h} \mid A \in \Theta^{ \pm}(n)_{h}, \bar{\lambda} \in\left(\mathbb{Z}_{l^{\prime} p^{h-1}}\right)^{n}\right\}$. According to [12, 4.8], $\widetilde{\mathrm{u}}_{k}(n, r)_{h}$ is a $\mathcal{k}$-subalgebra of $\mathcal{S}_{k}(n, r)$. Note that the algebra $\widetilde{\mathrm{u}}_{k}(n, r)_{1}$ is the little $q$-Schur algebra introduced in $[11,14]$. We will prove in 6.1 that the algebra $\widetilde{\mathrm{u}}_{k}(n, r)_{h}$ is a homomorphic image of $\widetilde{\mathrm{u}}_{k}(n)_{h}$.

Let $\mathcal{S}_{\mathcal{Q}}(n, r)=\mathcal{S}_{\mathcal{Z}}(n, r) \otimes_{\mathcal{Z}} \mathbb{Q}(v)$. For $A \in \Theta^{ \pm}(n), \delta \in \mathbb{Z}^{n}$ let

$$
A(\delta, r)=\sum_{\mu \in \Lambda(n, r-\sigma(A))} v^{\mu . \delta}[A+\operatorname{diag}(\mu)] \in \mathcal{S}_{\mathcal{Q}}(n, r) .
$$

According to [1], there is an algebra epimorphism

$$
\zeta_{r}: U_{\mathcal{Q}}(n) \rightarrow \mathcal{S}_{\mathcal{Q}}(n, r)
$$

satisfying $\zeta_{r}\left(E_{i}\right)=E_{i, i+1}(\mathbf{0}, r), \zeta_{r}\left(K_{1}^{j_{1}} K_{2}^{j_{2}} \cdots K_{n}^{j_{n}}\right)=0(\mathbf{j}, r)$ and $\zeta_{r}\left(F_{i}\right)=$ $E_{i+1, i}(\mathbf{0}, r)$, for $1 \leqslant i \leqslant n-1$ and $\mathbf{j} \in \mathbb{Z}^{n}$. It is proved in [9] that $\zeta_{r}\left(U_{\mathcal{Z}}(n)\right)=$ $\mathcal{S}_{\mathcal{Z}}(n, r)$. By restriction, the map $\zeta_{r}: U_{\mathcal{Q}}(n) \rightarrow \mathcal{S}_{\mathcal{Q}}(n, r)$ induces a surjective algebra homomorphism $\zeta_{r}: U_{\mathcal{Z}}(n) \rightarrow \mathcal{S}_{\mathcal{Z}}(n, r)$. The map $\zeta_{r}: U_{\mathcal{Z}}(n) \rightarrow$ $\mathcal{S}_{\mathcal{Z}}(n, r)$ induces an algebra homomorphism

$$
\zeta_{r, k}:=\zeta_{r} \otimes i d: U_{k}(n) \rightarrow \mathcal{S}_{\kappa}(n, r)
$$

Proposition 6.1. If either $l^{\prime}$ is odd or both $l^{\prime}$ is even and $\mathcal{K}$ is a field then $\zeta_{r, k}\left(\widetilde{\mathrm{u}}_{\kappa}(n)_{h}\right)=\widetilde{\mathrm{u}}_{k}(n, r)_{h}$. 
Proof. According to $[10,6.7]$, there is a surjective algebra homomorphism

$$
\dot{\zeta}_{r}: \mathcal{K}_{\mathcal{Z}}(n) \rightarrow \mathcal{S}_{\mathcal{Z}}(n, r)
$$

such that

$$
\dot{\zeta}_{r}([A])= \begin{cases}{[A]} & \text { if } A \in \Theta(n, r) ; \\ 0 & \text { otherwise. }\end{cases}
$$

The map $\dot{\zeta}_{r}$ induces a surjective algebra homomorphism

$$
\widehat{\dot{\zeta}}_{r, \kappa}: \widehat{\mathcal{K}}_{k}(n) \rightarrow \mathcal{S}_{k}(n, r)
$$

defined by sending $\sum_{A \in \widetilde{\Theta}(n)} \beta_{A}[A]_{\varepsilon}$ to $\sum_{A \in \Theta(n, r)} \beta_{A}[A]_{\varepsilon}$. It is easy to see that

$$
\zeta_{r, k}=\widehat{\dot{\zeta}}_{r, k} \circ \xi
$$

where $\xi$ is given in (5.0.1). This together with (5.0.2) implies that $\zeta_{r, k}\left(\widetilde{\mathrm{u}}_{k}(n)_{h}\right)$ $=\widehat{\dot{\zeta}}_{r, \kappa}\left(\mathcal{V}_{k}(n)_{h}\right)$. Clearly, for $A \in \Theta^{ \pm}(n)_{h}$ and $\bar{\lambda} \in\left(\mathbb{Z}_{l^{\prime} p^{h-1}}\right)^{n}$, we have $\hat{\dot{\zeta}}_{r, k}(\llbracket A+$ $\left.\operatorname{diag}(\bar{\lambda}) \rrbracket_{h}\right)=\llbracket A+\operatorname{diag}(\bar{\lambda}), r \rrbracket_{h}$. Combining these facts with 5.1 and 5.3 gives the result.

Let $\mathbf{s}_{k}(n, r)_{h}$ be the the infinitesimal $q$-Schur algebra introduced in $[2$, 3]. The algebra $\mathbf{s}_{\kappa}(n, r)_{h}$ is a certain $\mathcal{k}$-subalgebra of the $q$-Schur algebra $\mathcal{S}_{k}(n, r)$. According to $[2,5.3 .1]$, we have the following result.

Lemma 6.2. The set $\left\{[A]_{\varepsilon} \mid A \in \Theta(n, r)_{h}\right\}$ forms a $k$-basis of $\mathbf{s}_{k}(n, r)_{h}$.

For $h \geqslant 1$ let $\mathbf{s}_{k}(n)_{h}$ be the $k$-subalgebra of $U_{k}(n)$ generated by the elements $E_{i}^{(m)}, F_{i}^{(m)}, K_{j}^{ \pm 1},\left[\begin{array}{c}K_{j} ; 0 \\ t\end{array}\right]$ for $1 \leqslant i \leqslant n-1,1 \leqslant j \leqslant n, t \in \mathbb{N}$ and $0 \leqslant m<l p^{h-1}$. We will prove in 6.4 that the algebra $\mathbf{s}_{k}(n, r)_{h}$ is a homomorphic image of $\mathbf{s}_{k}(n)_{h}$.

Lemma 6.3. Each of the following set forms a $\mathcal{K}$-basis for $\mathbf{s}_{\kappa}(n)_{h}$ :

(1) $\left\{E^{\left(A^{+}\right)} \prod_{1 \leqslant i \leqslant n} K_{i}^{\delta_{i}}\left[\begin{array}{c}K_{i} ; 0 \\ \lambda_{i}\end{array}\right] F^{\left(A^{-}\right)} \mid A \in \Theta^{ \pm}(n)_{h}, \delta \in \mathbb{N}^{n}, \delta_{i} \in\{0,1\}\right.$, $\left.\forall i, \lambda \in \mathbb{N}^{n}\right\}$

(2) $\left\{A(\delta, \lambda) \mid A \in \Theta^{ \pm}(n)_{h}, \delta, \lambda \in \mathbb{N}^{n}, \delta_{i} \in\{0,1\}, \forall i\right\}$.

Proof. The assertion can be proved in a way similar to the proof of 3.7.

Proposition 6.4. We have $\zeta_{r, k}\left(\mathbf{s}_{k}(n)_{h}\right)=\mathbf{s}_{k}(n, r)_{h}$. 
Proof. From 6.1.1 we see that

$$
\zeta_{r, \kappa}(A(\delta, \lambda))=\widehat{\dot{\zeta}}_{r, \kappa}\left(A(\delta, \lambda)_{\varepsilon}\right)=A(\delta, \lambda, r)_{\varepsilon}
$$

for all $A, \delta, \lambda$, where $A(\delta, \lambda, r)_{\varepsilon}=\sum_{\mu \in \Lambda(n, r-\sigma(A))} \varepsilon^{\mu . \delta}\left[\begin{array}{c}\mu \\ \lambda\end{array}\right]_{\varepsilon}[A+\operatorname{diag}(\mu)]_{\varepsilon} \in$ $\mathcal{S}_{k}(n, r)$. Thus by 6.2 and 6.4 we conclude that

$$
\begin{aligned}
\zeta_{r, \kappa}\left(\mathbf{s}_{\kappa}(n)_{h}\right) & =\operatorname{span}_{\kappa}\left\{A(\delta, \lambda, r)_{\varepsilon} \mid A \in \Theta^{ \pm}(n)_{h}, \delta, \lambda \in \mathbb{N}^{n}, \delta_{i} \in\{0,1\}, \forall i\right\} \\
& \subseteq \mathbf{s}_{\mathcal{K}}(n, r)_{h} .
\end{aligned}
$$

On the other hand, for $A \in \Theta^{ \pm}(n)_{h}$ and $\mu \in \Lambda(n, r-\sigma(A))$ we have $[A+$ $\operatorname{diag}(\mu)]=A(\mathbf{0}, \mu, r) \in \zeta_{r, k}\left(\mathbf{s}_{\mathcal{K}}(n)_{h}\right)$. This implies that $\mathbf{s}_{\mathcal{K}}(n, r)_{h} \subseteq \zeta_{r, k}\left(\mathbf{s}_{\kappa}(n)_{h}\right)$. The assertion follows.

\section{Acknowledgements}

The author was supported by the National Natural Science Foundation of China, the Program NCET, Fok Ying Tung Education Foundation and the Fundamental Research Funds for the Central Universities.

\section{References}

[1] A. A. Beilinson, G. Lusztig and R. MacPherson, A geometric setting for the quantum deformation of $G L_{n}$, Duke Math. J. 61 (1990), 655-677.

[2] A. G. Cox, On some applications of infinitesimal methods to quantum groups and related algebras, Ph.D thesis, University of London, 1997.

[3] A. G. Cox, On the blocks of the infinitesimal Schur algebras, Quart. J. Math 51 (2000), 39-56.

[4] R. Dipper and G. James, The q-Schur algebra, Proc. London Math. Soc. 59 (1989), 23-50.

[5] R. Dipper and G. James, q-Tensor spaces and $q$-Weyl modules, Trans. Amer. Math. Soc. 327 (1991), 251-282.

[6] S. R. Doty, D. K. Nakano, and K. M. Peters, On Infinitesimal Schur algebras, Proc. London Math. Soc. 72 (1996), 588-612.

[7] C. M. Drupieski, Representations and cohomology for Frobenius-Lusztig kernels, J. Pure Appl. Algebra 215 (2011), 1473-1491. 
[8] J. Du, Kazhdan-Lusztig bases and isomorphism theorem for q-Schur algebras, Comtemp. Math. 139 (1992), 121-140.

[9] J. Du, A note on the quantized Weyl reciprocity at roots of unity, Alg. Colloq. 2(1995), 363-372.

[10] J. Du and Q. Fu, Quantum $\mathfrak{g l}_{\infty}$, infinite q-Schur algebras and their representations, J. Algebra 322 (2009), 1516-1547.

[11] J. Du, Q. Fu, and J.-P. Wang, Infinitesimal quantum $\mathfrak{g l}_{n}$ and little qSchur algebras, J. Algebra 287 (2005), 199-233.

[12] J. Du, Q. Fu, and J.-P. Wang, Representations of little q-Schur algebras, Pacific J. Math. 257(2012), 343-378.

[13] Q. Fu, A comparison between little and infinitesimal q-Schur algebras, Comm. Algebra, 33 (2005), 2663-2682.

[14] Q. Fu, Little q-Schur algebras at even roots of unity, J. Algebra, 311(2007), 202-215.

[15] Q. Fu, Affine quantum Schur algebras at roots of unity, preprint, arXiv:1205. 2997.

[16] Q. Fu, BLM realization for the integral form of quantum $\mathfrak{g l}_{n}$, Commun. Contemp. Math. 17 (2015), 1550019, 17 pp.

[17] M. Jimbo, A q-analogue of $U(\mathfrak{g l}(N+1))$, Hecke algebras, and the YangBaxter equation, Lett. Math. Phy. 11 (1986), 247-252.

[18] G. Lusztig, Modular representations and quantum groups, Comtemp. Math. 82 (1989), 59-77.

[19] G. Lusztig, Finite dimensional Hopf algebras arising from quantized universal enveloping algebras, J. Amer. Math. Soc. 3 (1990), 257-296.

[20] G. Lusztig, Canonical bases arising from quantized enveloping algebras, J. Amer. Math. Soc. 3 (1990), 447-498.

[21] C. M. Ringel, Hall algebras and quantum groups, Invent. Math. 101 (1990), 583-592. 
School of Mathematical Sciences, Tongui University Shanghai, 200092, China

E-mail address: q.fu@hotmail.com, q.fu@tongji.edu.cn

ReCeIVed DeCEMBer 25, 2013 